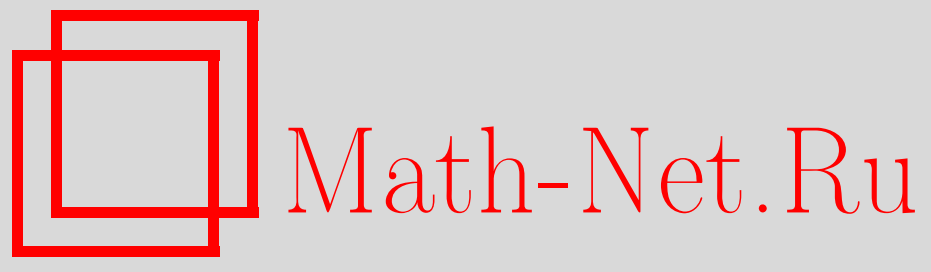

А. Н. Паршин, Вопросы и замечания к программе Ленглендса, УМН, 2012, том 67, выпуск 3, 115-146

DOI: https://doi.org/10.4213/rm9479

Использование Общероссийского математического портала Math-Net.Ru подразумевает, что вы прочитали и согласны с пользовательским соглашением http: //www . mathnet.ru/rus/agreement

Параметры загрузки:

IP: 18.207 .199 .55

26 апреля 2023 г., 05:38:17

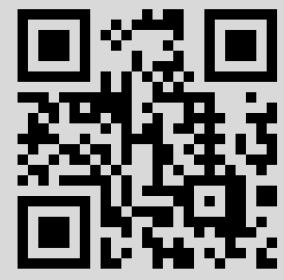




\section{Вопросы и замечания к программе Ленглендса}

\section{А. Н. Паршин}

Дается краткий обзор классической программы Ленглендса построения соответствия между $n$-мерными представлениями групп Галуа локальных и глобальных полей размерности 1 и неприводимыми представлениями групп $\mathrm{GL}(n)$, связанных с такими полями и их кольцами аделей. Рассмотрено обобщение программы Лендглендса на случай полей размерности 2 и описан его вариант для одномерных представлений. Сформулирована гипотеза о прямом образе автоморфных форм, связывающая соответствия Ленглендса в размерностях 2 и 1 . Показано, что в геометрическом случае поверхностей над конечным полем она вытекает из теоремы Лаффорга о существовании глобального соответствия Ленглендса для кривых. Из гипотезы о прямом образе вытекает также классическая гипотеза Хассе-Вейля об аналитическом поведении дзета- и $L$-функций кривых, определенных над глобальными полями размерности 1.

Библиография: 56 названий.

Ключевые слова: соответствие Ленглендса, автоморфные формы, $L$-функции, двумерные локальные поля, адели, $K$-группы, теория полей классов, прямые образы.

\section{СОДЕРЖАНИЕ}

1. Введение........................................... 116

2. Основные поля с точки зрения теории схем.................... 120

3. Двумерное обобщение соответствия Ленглендса ................... 123

4. Функториальные свойства соответствия Ленглендса................ 124

5. Отношение к геометрическому соответствию Дринфельда-Ленглендса 128

6. Гипотеза о прямом образе............................... 132

7. Связь с гипотезой Хассе-Вейля . . . . . . . . . . . . . . . . . . 138

8. Приложение. Нульмерное обобщение соответствия Ленглендса........ 140

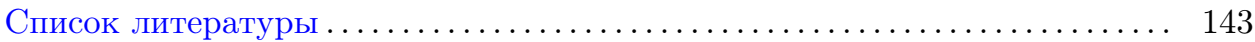

Я признателен Р. П. Ленглендсу за очень полезные для меня обсуждения во время его визита в МИАН (Москва, октябрь 2011 г.), Майклу Харрису и Ульриху Штулеру, которые отвечали на мои подчас слишком наивные вопросы, и Ильхану Икеда, прочитавшему первый вариант текста и сделавшему ряд замечаний. Работа была поддержана грантами РФФИ 1101.00145-а, 1101.12098-офи-м и НШ-5139.2012.1. 


\section{1. Введение}

Программа Ленглендса состоит в установлении соответствия между представлениями групп Галуа (и их обобщений) и представлениями редуктивных алгебраических групп. Исходным объектом является некоторое поле. Рассматриваются шесть типов полей: три типа локальных полей и три типа глобальных полей [35], [14]. К первым относятся:

1) конечные расширения полей $p$-адических чисел $\mathbb{Q}_{p}$, поле $\mathbb{R}$ вещественных чисел и поле $\mathbb{C}$ комплексных чисел,

2) поля степенных рядов Лорана $\mathbb{F}_{q}((t))$, где $\mathbb{F}_{q}$ - конечное поле из $q$ элементов,

3) поле степенных рядов Лорана $\mathbb{C}((t))$.

Глобальные поля - это:

4) поля алгебраических чисел (= конечные расширения поля $\mathbb{Q}$ рациональных чисел),

5) поля алгебраических функций от одной переменной с конечным полем констант (= конечные расширения поля рациональных функций $\mathbb{F}_{q}(x)$ ),

6) поля алгебраических функций от одной переменной с полем констант $\mathbb{C}$ (= конечные расширения поля $\mathbb{C}(x))$.

При этом локальные поля из первого списка выступают как пополнения глобальных полей из второго.

В классической программе Ленглендса рассматриваются только первые два типа локальных и глобальных полей. Программа, в своем наиболее простом (и изученном) варианте, состоит в построении соответствия между конечномерными (размерности $n)$ представлениями группы Галуа $G_{K}=\operatorname{Gal}\left(K^{\mathrm{sep}} / K\right)$ сепарабельного замыкания рассматриваемого поля $K$ и неприводимыми бесконечномерными (как правило) представлениями группы $\mathrm{GL}\left(n, A_{K}\right)$, где $A_{K}=K$ для локальных полей $K$ и $A_{K}=\mathbb{A}_{K}$ (кольцо аделей поля $K$ ) для глобальных полей $K$.

Напомним, что $\mathbb{A}_{K}=\left\{\left(f_{v}\right): f_{v} \in K_{v}\right.$ для всех $v, f_{v} \in \widehat{\mathscr{O}}_{v}$ для почти всех $\left.v\right\}$. При этом $v$ пробегает все классы нормирований поля $K$ с полными кольцами нормирования $\widehat{\mathscr{O}}_{v}$ и локальными полями $K_{v}=\operatorname{Frac}\left(\widehat{\mathscr{O}}_{v}\right) \supset K$ (локальными пополнениями поля $K$ ). В числовом случае сюда надо добавить конечное число архимедовых нормирований поля $K$ и соответствующих его вложений в поля $\mathbb{R}$ или $\mathbb{C}$.

В глобальном случае рассматриваемые представления должны быть автоморфными, т. е. реализуемыми в пространстве функций на факторпространстве $\operatorname{GL}\left(n, \mathbb{A}_{K}\right) / \mathrm{GL}(n, K)$. Поскольку $n$-мерные представления группы Галуа $G_{K}$ можно рассматривать как гомоморфизмы из $G_{K}$ в $\mathrm{GL}(n, \mathbb{C})$, то возникает естественное обобщение этого соответствия, когда группа $\mathrm{GL}(n)$ заменяется произвольной редуктивной алгебраической группой $G$. И в этой более общей ситуации гипотетическое соответствие Ленглендса должно выглядеть (в первом приближении) так:

$$
\operatorname{Hom}\left(G_{K},{ }^{L} G(\mathbb{C})\right) \Leftrightarrow \quad \begin{aligned}
& \text { неприводимые (автоморфные) } \\
& \text { представления группы } G\left(A_{K}\right) .
\end{aligned}
$$


Здесь ${ }^{L} G$ - редуктивная алгебраическая группа, двойственная по Ленглендсу группе $G$ (см. определение и основные свойства в [51], [8]). Важным и весьма нетривиальным является то, что если исходная группа $G$ рассматривается как редуктивная группа над различными полями из основного списка, то двойственная группа ${ }^{L} G$ всегда является группой, определенной над полем комплексных чисел ${ }^{1}$. Группа ${ }^{L} G$ содержит связную компоненту единицы ${ }^{L} G_{0}$ (редуктивную алгебраическую группу). Ее корневая система строится с помощью двойственности торов, исходя из корневой системы исходной группы $G$. При этом для общей линейной группы $G=\mathrm{GL}(n)$ корневые системы групп $G$ ${ }_{\text {и }}{ }^{L} G$ совпадают. Так что в этом случае наличие двойственности является замаскированным. Наконец, вся группа ${ }^{L} G$ является полупрямым произведением групп ${ }^{L} G_{0}$ и группы Галуа $\operatorname{Gal}\left(K^{\text {sep }} / K\right)$.

Представления групп Галуа, входящие в соответствие Ленглендса "слева", нуждаются в существенном обобщении, чтобы можно было надеяться на существование биекции с автоморфными представлениями редуктивных групп, находящимися "справа" 2 . В теории $L$-рядов Артина рассматриваются представления конечных групп Галуа со значениями в группе $\operatorname{GL}(n, \mathbb{C})$. Если перейти к группе Галуа $G_{K}$ сепарабельного замыкания основного поля $K$, то все ее непрерывные представления в группе $\mathrm{GL}(n, \mathbb{C})$ получаются из представлений конечных факторов $\operatorname{Gal}(L / K)$, где $L / K$ пробегает все нормальные конечные расширения поля $K$. Таким образом, непрерывных комплексных представлений групп Галуа не так много. С другой стороны, есть непрерывные $l$-адические представления, возникающие из действия группы Галуа на этальные когомологии алгебраических многообразий, определенных над $K$. Они, как правило, не пропускаются через конечные факторы группы Галуа ${ }^{3}$. Делинь придумал, как построить некоторое расширение группы Галуа $G_{K}$, у которого комплексные представления "совпадали бы” с $l$-адическими представлениями группы $G_{K}$. Группа Вейля-Делиня $W D_{K}$ является полупрямым расширением группы Вейля ${ }^{4} W_{K}$ с помощью группы $\mathbb{C}$ и действия $w z w^{-1}=q^{-\nu(w)} z$. Именно,

$$
W D_{K}=\left\{w, z: w \in W_{K}, z \in \mathbb{C}\right\}
$$

и

$$
(w, z) \cdot\left(w^{\prime}, z^{\prime}\right)=\left(w w^{\prime}, z+q^{-\nu(w)} z^{\prime}\right),
$$

где $\nu: W_{K} \rightarrow \mathbb{Z}$ - каноническое отображение группы Вейля, переводящее $n$-ю степень $Ф$ робениуса в $n$, и $q$ - число элементов в поле вычетов ${ }^{5}$.

\footnotetext{
${ }^{1}$ Или над другим полем, например $\overline{\mathbb{Q}}_{l}$, которое не имеет ничего общего с основным полем $K$.

2 Заметим, что наличие биекции предполагается лишь для группы $G=\mathrm{GL}(n)$. В общем случае соответствие может не быть взаимно однозначным, а иметь в качестве прообразов конечные множества, так называемые $L$-пакеты.

ЗЭто верно, если все циклы на многообразии являются алгебраическими. В силу гипотез Тейта должно быть верно и обратное.

${ }^{4}$ Группа Вейля $W_{K}$ локального поля $K$ с конечным полем вычетов является подгруппой группы Галуа $G_{K}$, состоящей из тех ее элементов, которые действуют на поле вычетов целыми степенями автоморфизма Фробениуса.

${ }^{5}$ Детали см. в [51; $\left.\$ 4.1\right]$. Вместо группы Вейля-Делиня можно рассматривать представления группы Вейля-Артура $W A_{K}=W_{K} \times \mathrm{SL}(2, \mathbb{C})$. Переход от представлений одной группы к представлениям другой обеспечивается теоремой Джекобсона-Морозова (см. [35]).
} 
В частности, соответствие Ленглендса $\mathrm{LC}_{n}$ для локальных полей и группы $\mathrm{GL}(n)$ должно иметь вид

$$
\operatorname{Hom}^{\mathrm{c}, \mathrm{ss}}\left(W D_{K}, \mathrm{GL}(n, \mathbb{C})\right) \Leftrightarrow \quad \begin{aligned}
& \text { гладкие неприводимые } \\
& \text { представления группы } \operatorname{GL}(n, K),
\end{aligned}
$$

и это уже окончательная точная формулировка (представления группы ВейляДелиня предполагаются непрерывными и вполне приводимыми). Гладкость представления $\pi: \operatorname{GL}(n, K) \rightarrow \operatorname{End}(V)$ означает, что для любого $v \in V$ найдется такая открытая компактная подгруппа $\mathbb{K}^{\prime}$ в $\mathrm{GL}(n, K)$, что $\pi\left(\mathbb{K}^{\prime}\right)(v)=v$. Неприводимость означает отсутствие любых нетривиальных инвариантных подпространств в $V$. Пусть $\rho, \rho^{\prime}$ пробегают $n$-мерные представления группы Вейля-Делиня, а $\pi$ - представления группы $\mathrm{GL}(n, K)$. Соответствие Ленглендса обладает следующими свойствами:

i) $\mathrm{LC}_{1}(\operatorname{Det} \rho)=$ центральный характер представления $\mathrm{LC}_{n}(\rho)$;

ii) $\mathrm{LC}_{n}(\rho \otimes \chi)=\mathrm{LC}_{n}(\rho) \otimes\left(\mathrm{LC}_{1}(\chi) \circ \mathrm{Det}\right)$, где $\chi$ - одномерное представление;

iii) $\operatorname{LC}_{m+n}\left(\rho \times \rho^{\prime}\right)=\operatorname{Ind}_{P}^{\mathrm{GL}(m+n, K)} \circ \operatorname{Res}_{M}^{P}\left[\operatorname{LC}_{m}(\rho) \otimes \operatorname{LC}_{n}\left(\rho^{\prime}\right)\right]$;

iv) если представление $\rho$ неприводимо, то представление $\operatorname{LC}_{n}(\rho)$ является каспидальным;

v) $\mathrm{LC}_{n}(\check{\rho})=\check{L C C}_{n}(\rho)$;

vi) $L(\rho, s)=L\left(\mathrm{LC}_{n}(\rho), s\right)$;

vii) если представление $\rho$ неразветвлено, то представление $\mathrm{LC}_{n}(\rho)$ является сферическим.

В свойстве iii) использовано вложение групп $\operatorname{GL}(m, K)$ и $\operatorname{GL}(n, K)$ в группу $\mathrm{GL}(m+n, K)$ в качестве фактора Леви $M=\mathrm{GL}(m, K) \times \mathrm{GL}(n, K)$ стандартной параболической подгруппы $P$. Об операции $\otimes$ см. сноску 17 . Последующая операция параболической индукиии состоит в подъеме представления на подгруппу $P$ и затем индуцирования на всю группу $\mathrm{GL}(m+n, K)$ (подробности, а также определение каспидального представления см. [4], [7] и [6; гл. 4] для случая GL(2)). Далее, $\check{\rho}(\check{\pi})$ - двойственное (контраградиентное) представлению $\rho(\pi)$, а $L(\rho, s)$ и $L(\pi, s)-L$-функции представлений $\rho$ и представления $\pi$ (см. раздел 7). Представление $\pi$ группы $\operatorname{GL}(n, K)$ называется сферическим, если в пространстве $V$ представления существует такой вектор $v \in V$, что $\pi(\mathbb{K})(v)=v$, где $\mathbb{K}=\operatorname{GL}\left(n, \mathscr{O}_{K}\right)$ - максимальная компактная подгруппа. Список свойств можно еще дополнить равенством для локальных констант, входящих в функциональные уравнения $L$-функций (подробнее см. [20]).

Аналогичные свойства должны иметь место и для глобального соответствия Ленглендса.

В настоящее время соответствие Ленглендса построено для локальных полей типа 1) (M. Harris-R. Taylor [18]; G. Henniart [20]) и 2) (G. Laumon-M. Rapoport-U. Stuhler [36]) именно в такой форме. Для глобальных полей типа 5) и группы $G=\mathrm{GL}(n)$ оно получено В. Г. Дринфельдом [9], [10] для $n=2$ и Л. Лаффоргом [32] для произвольного $n$. В числовом случае конструкция соответствия остается открытым вопросом и, видимо, очень далеким от разрешения. 
Предыдущие конструкции имеют чисто арифметическое происхождение. Исходя из своего доказательства, где основную роль играют пространства модулей векторных расслоений на алгебраических кривых, Дринфельд расширил класс рассматриваемых полей и ввел геометрическое соответствие Ленглендса, которое должно иметь место для полей типа 3) и 6).

Исходным объектом тут выступает полная алгебраическая кривая $X$, определенная над полем $\mathbb{C}$ и для которой поле $K$ служит полем рациональных функций. Соответствие выглядит следующим образом:

локальные ${ }^{L} G$-системы на $X \Leftrightarrow$ пучки Гекке на схеме (stack'e) $\operatorname{Bun}_{X, G}$

и доказано для неразветвленных локальных систем и группы $G=\operatorname{GL}(n)$ (В. Г. Дринфельд для $n=2$ и E. Frenkel-D. Gaitsgory-K. Vilonen для любого $n$, основываясь на конструкции Laumon'a). Локальные (неразветвленные) ${ }^{L} G$-системы на $X$ суть не что иное, как представления фундаментальной группы кривой $X$ в группе ${ }^{L} G$, что выглядит вполне естественным аналогом арифметической ситуации. С другой стороны, имеется $\operatorname{Bun}_{X, G}-\operatorname{stack}^{6}$ главных $G$-расслоений на $X$ и на нем действует кольцо соответствий Гекке. Необходимые пучки на $\operatorname{Bun}_{X, G}$, которые рассматриваются вместо автоморфных форм в классическом соответствии Ленглендса, - это perverse пучки, заменяющие обычные локальные системы. Такой пучок называется пучком Гекке, если он полуинвариантен относительно соответствий Гекке. В этой ситуации пучки Гекке не заменяют представления группы аделей, отвечающие согласно арифметическому соответствию Ленглендса представлениям группы Галуа, а соответствуют сферическим векторам этих представлений. Имеются ли сами представления, я не знаю, но если они есть, то должны быть в разумном смысле также неразветвленными (сферическими). В арифметической ситуации сферический вектор определен однозначно, с точностью до константы, и порождает все пространство представления.

Какие задачи теории чисел можно изучать с помощью программы Ленглендca?

1. Неабелева теория полей классов. Соответствие Ленглендса возникло из рассмотрения абелевой ситуации, когда $G=\mathrm{GL}(1)$ и представления группы Галуа суть гомоморфизмы группы $G_{K}$ в $\mathrm{GL}(1, \mathbb{C})=\mathbb{C}^{*}$, т. е. характеры группы $G_{K}$. Раньше такие отображения называли квазихарактерами, удерживая термин "характер" для гомоморфизмов в унитарную группу $U(1) \subset \mathrm{GL}(1, \mathbb{C})$. В силу теории полей классов имеем отображение взаимности

$$
C_{K} \rightarrow G_{K}^{\mathrm{ab}}
$$

где $C_{K}=K^{*}$ для локальных полей типа 1) и 2) и $C_{K}=\mathbb{A}_{K} / K^{*}$ для глобальных полей типов 4) и 5). Характеры группы $C_{K}$ (и они суть автоморфные формы на $\mathrm{GL}(1))$ переносятся на группу $G_{K}$ в виде ее абелевых характеров, т. е. 1-мерных представлений (или их прямых сумм). Мы получаем таким образом абелево соответствие Ленглендса [34], которое и послужило отправной точкой всей программы.

\footnotetext{
${ }^{6}$ Автор приложил довольно много усилий, чтобы найти достойный русский эквивалент этого слова, но не преуспел в этом деянии.
} 
Имеется, однако, существенное отличие конструкции Ленглендса от абелева случая, именно с точки зрения описания группы Галуа. Теория полей классов позволяет явно вычислить группу Галуа максимального абелева расширения исходного поля (задавая ее образующими и соотношениями). И, более того, когомологическая формулировка теории полей классов дает возможность вычислять группы Галуа некоторых неабелевых расширений (l-расширений [49], [30]). Программа Ленглендса дает возможность описать множество представлений группы Галуа, снабдив его набором стандартных операций (прежде всего прямым и тензорным произведениями). Дальнейшее развитие программы (см. обсуждение в [35], [44]) состояло в предложении построить категорию представлений группы Галуа как моноидальную категорию (более точно, как категорию Таннака или, скорее, ее обобщение), что позволяет в силу общей теоремы о категориях Таннака однозначно восстановить группу [5]. Заметим, что эта теорема есть теорема существования и неясно, как получить явное представление группы образующими и соотношениями.

2. Гипотезы о дзета- и L-функииях арифметических схем (см. ниже раздел 7 о гипотезе Хассе-Вейля).

В этом направлении большим достижением является доказательство гипотезы Артина о целости $L$-рядов для широкого класса двумерных представлений группы Галуа поля $\mathbb{Q}(\mathrm{R} . \mathrm{P}$. Langlands-J. Tunnell). Так, частный случай октаэдральных представлений был отправной точкой работы А. Вайлса по доказательству гипотезы Танияма-Вейля.

3. Описание множества гладких неприводимых представлений редуктивных групп над локальными и глобальными полями.

Последнее направление является наиболее успешным. Мы видели, как точка зрения теории представлений редуктивных групп диктует необходимость замены группы Галуа поля на группу Вейля-Делиня или группу Вейля-Артура. Наиболее ярко это проявляется на примере локального поля $K=\mathbb{C}$. Группа $\mathrm{GL}(n, K)$ имеет для $K=\mathbb{C}$ нетривиальное множество неприводимых представлений (все они являются представлениями основной серии; их пространство параметров является комплексным многообразием, а именно, фактором многообразия характеров тора $\left(\mathbb{C}^{*}\right)^{n}$ по группе перестановок $\left.{ }^{7} S_{n}\right)$. В то же время группа Галуа $G_{K}$ тривиальна, но группа Вейля согласно общей теории [2; гл. XV], $[51 ; \S 1]$ равна $W_{\mathbb{C}}=\mathbb{C}^{*}$ и имеет ровно такое же пространство $n$-мерных полупростых представлений.

Мы рассмотрим теперь основные поля программы Ленглендса с точки зрения общих принципов арифметической алгебраической геометрии.

\section{2. Основные поля с точки зрения теории схем}

Поля, расматривамые в теории Ленглендса, являются полями функций на некоторых схемах. В теории схем мы имеем классификацию по размерности. Если схема аффинна и отвечает кольцу $A$, то можно рассмотреть его размерность по Круллю. Имеем такую таблицу колец, возникающих в арифметике:

\footnotetext{
${ }^{7}$ Теорема Д. П. Желобенко и М. А. Наймарка (1966, см. изложение в [56]). Явный вид характеров тора $\mathbb{C}^{*}$ и, следовательно, $\left(\mathbb{C}^{*}\right)^{n}$ см. ниже, а также подробнее в [29].
} 


\begin{tabular}{|c|c|c|}
\hline $\operatorname{Dim}(A)$ & геометрический случай & числовой случай \\
\hline$>2$ & $\cdots$ & $\cdots$ \\
\hline 2 & $\begin{array}{c}\mathbb{F}_{q}[x, y] \\
\text { (поверхности) }\end{array}$ & $\begin{array}{l}\mathbb{Z}[y] \\
\text { (арифметические поверхности) }\end{array}$ \\
\hline 1 & $\begin{array}{c}\mathbb{F}_{q}[x] \\
\text { кривые) }\end{array}$ & $\begin{array}{l}\mathbb{Z} \\
\text { (арифметические кривые, } \operatorname{Spec}(\mathbb{Z}))\end{array}$ \\
\hline 0 & $\mathbb{F}_{q}$ & $\mathbb{F}_{1}$ \\
\hline
\end{tabular}

Здесь $\mathbb{F}_{q}-$ конечное поле из $q$ элементов, $\mathbb{Z}-$ кольцо целых чисел и $\mathbb{F}_{1}-$ популярное теперь поле из одного элемента.

Этим кольцам отвечают аффинные схемы и поля функций на них. В частности, сюда входят глобальные поля типов 4) и 5) из списка полей, которые теперь рассматриваются в качестве основных для программы Ленглендса [35], [14]:

\begin{tabular}{|c|c|c|}
\hline $\operatorname{Dim}(A)$ & геометрический случай & числовой случай \\
\hline 2 & $\begin{array}{c}\mathbb{F}_{q}(x, y) \\
\text { (рациональные функции) }\end{array}$ & $\begin{array}{c}\mathbb{Q}(y) \\
\text { (рациональные функции) }\end{array}$ \\
\hline 1 & $\mathbb{F}_{q}(x)$ & $\mathbb{Q}$ \\
(рациональные функции) & (рациональные числа) \\
\hline 0 & $\mathbb{F}_{q}$ & $\mathbb{F}_{1}$ \\
\hline
\end{tabular}

Наконец, есть таблица возникающих в этой ситуации локальных полей:

\begin{tabular}{|c|c|c|}
\hline $\operatorname{Dim}(A)$ & геометрический случай & числовой случай \\
\hline \multirow{2}{*}{2} & $\mathbb{F}_{q}((x))((y))$ & $\mathbb{Q}_{p}((y)), \mathbb{R}((y)), \mathbb{C}((y))$ \\
& $\begin{array}{c}\text { (итерированные степенные } \\
\text { ряды Лорана) }\end{array}$ & ряды Лореннана $)$ \\
\hline \multirow{2}{*}{1} & $\mathbb{F}_{q}((x))$ & $\mathbb{Q}_{p}, \mathbb{R}, \mathbb{C}$ \\
& (степенные & $(p$-адические, вещественные \\
& ряды Лорана) & и комплексные числа $))$ \\
\hline
\end{tabular}

Здесь мы видим все типы 1)-3) локальных полей, но и целый ряд новых полей, которые были введены в высшей адельной теории $[42]^{8}$. Подобная классификация является развитием идей доклада Андре Вейля на конгрессе 1950 г. в Кембридже [53], где он подчеркнул важность понятия размерности по Круллю для классификации арифметических задач. В его довоенном письме сестре Симоне [52], где Вейль тоже говорит об аналогии между числовым и геометрическим случаями, ситуация упрощена и поэтому он добавляет поле $\mathbb{C}((y))$ и ставит его на "одну доску" с такими полями, как $\mathbb{F}_{q}((x)), \mathbb{Q}_{p}$, что, как видно из таблицы, не согласуется с размерностью.

Возвращаясь к глобальным полям, можно спросить, где в этих таблицах такие схемы, как кривые над полем комплексных чисел, связанные с полями типа 6)? Эти кривые и поля функций на них суть предмет геометрического соответствия Ленглендса, обобщающего обычное соответствие Ленглендса.

\footnotetext{
${ }^{8}$ См. неформальное определение в начале следующего раздела.
} 
Их, действительно, тут нет, так как они отвечают кольцам вида $\mathbb{C}[x]$. Такие объекты имеют промежуточную природу, они глобальны и размерности 1 по $x$ (это координата на кривой) и локальны по полю определения $\mathbb{C}$. Это поле входит в таблицу локальных полей как одномерное (!) поле. Таким образом, такие кольца, как $\mathbb{C}[x]$, соответствуют геометрическим объектам размерности 2. Именно, они суть архимедовы слои арифметических поверхностей (о них см. ниже в разделе 5).

Это замечание и имеющиеся в арифметике аналогии [41] предполагают рассмотрение, в качестве "партнера" геометрического соответствия Ленглендса для кривых над $\mathbb{C}$, некоторой конструкции такого же рода для кривых над полем $p$-адических чисел $\mathbb{Q}_{p}$. Насколько я знаю, это никем не рассматривалось, и далее мы обсудим, как это может выглядеть ${ }^{9}$.

Резюмируя это обсуждение, можно сказать, что список шести основных полей должен быть дополнен следующим образом. Лишь четыре типа полей 1), 2), 4) и 5) - относятся к одномерной ситуации. Поля типа 3) и 6) относятся уже к двумерной ситуации. В этом случае имеем два типа локальных полей:

1) конечные расширения полей $\mathbb{Q}_{p}((t)), \mathbb{R}((t)), \mathbb{C}((t))$ и $\mathbb{Q}_{p}\{\{t\}\}^{10}$,

2) поля степенных рядов Лорана $\mathbb{F}_{q}((u))((t))$,

и два типа глобальных полей:

3) поля функций на арифметических поверхностях (= конечные расширения поля $\mathbb{Q}(x)$ рациональных функций $)$,

4) поля функций на алгебраических поверхностях над конечным полем констант (= конечные расширения поля рациональных функций от двух переменных $\left.\mathbb{F}_{q}(x, y)\right)$.

Между ними располагаются поля промежуточных типов (частично локальных, частично глобальных). В ситуации поверхности над конечным полем промежуточными полями будут

$2 \mathrm{a})$ конечные расширения полей $\mathbb{F}_{q}(u)((t))$,

$2 \mathrm{~b})$ поля отношений кольца $\mathbb{F}_{q}[[u, t]]$ формальных степенных рядов от двух переменных.

В случае арифметических поверхностей сюда можно отнести и поле $\mathbb{C}(x)$ (это поле 6) из списка Ленглендса), но оно не входит в стандартный набор полей, возникающий в двумерной адельной теории (см. раздел 3 ниже и [42]), поскольку связано с дополнительной структурой: наличием морфизма рассматриваемой двумерной схемы на одномерную схему $B$ и его слоев, определенных над одномерными локальными полями схемы $B$.

ЗАмЕчАниЕ 1. Адельная техника позволяет рассматривать локальные и глобальные поля исходной программы Ленглендса единым образом. Как мы видели, в обоих случаях эти поля являются полями функций на некоторой схеме $X$.

\footnotetext{
${ }^{9}$ Как сообщил мне Ильхан Икеда, конструкция такого рода была недавно найдена А. Г. М. Паулином для абелева случая.

${ }^{10}$ Если $K$ - локально компактное поле с неархимедовой нормой $|\cdot|$, то поле $K\{\{t\}\}$ состоит из бесконечных в обе стороны рядов $\sum_{i} a_{i}, a_{i} \in K$ с $\left|a_{i}\right| \leqslant O(1),\left|a_{i}\right| \rightarrow 0$ для $i \rightarrow-\infty$. Поля $\mathbb{Q}_{p}\{\{t\}\}$ возникают на арифметических поверхностях.
} 
В локальном (неархимедовом!) случае имеем $X=\operatorname{Spec}(\mathscr{O})$, где $\mathscr{O}$ - локальное кольцо дискретного нормирования с конечным полем вычетов. В глобальной ситуации $X$ будет кривой над конечным полем или спектром кольца целых поля алгебраических чисел. И всегда $A_{K}=\mathbb{A}_{X}$ в смысле общего определения кольца аделей [11], [42], в которое можно включить и архимедовы компоненты.

\section{3. Двумерное обобщение соответствия Ленглендса}

Можно предположить существование двумерного обобщения соответствия Ленглендса. Пусть $X$ - двумерная схема и $K$ - поле функций на ней. Тогда представления размерности $n$ группы Галуа $G_{K}$ сепарабельного замыкания поля $K$ могли бы соответствовать неприводимым автоморфным представлениям группы аделей $G\left(\mathbb{A}_{X}\right)$. Кольцо аделей $\mathbb{A}_{X}$ было введено в высшей теории аделей в 1970-х годах (см. обзоры [11], [42]). Если $x \in C \subset X-$ флаг на $X$, состоящий из точки $x$ и неприводимой кривой $C$, то ему отвечает (если кривая $C$ гладка в $x$ ) двумерное локальное поле $K_{x, C}$. В локальных координатах $u, t$ формальной окрестности точки $x$, для которых $C=(t=0)$, поле $K_{x, C}$ равно $k(x)((u))((t))$, где $k(x)$ - поле вычетов точки $x$. В общем случае флагу отвечает конечная сумма двумерных локальных полей. Кольцо аделей $\mathbb{A}_{X}$ определяется как адельное (часть полного) произведение полей $K_{x, C}$ по всем флагам $x, C$.

Поле $K_{x, C}$ содержит кольцо дискретного нормирования $\widehat{\mathscr{O}}_{x, C}$ (которое есть $k(x)((u))[[t]]$, если $X$ - поверхность). Для неприводимой кривой $C$ положим $K_{C}=\operatorname{Frac}\left(\widehat{\mathscr{O}}_{C}\right)$, а для точки $x$ положим $K_{x}=K \widehat{\mathscr{O}}_{x} \subset \operatorname{Frac}\left(\widehat{\mathscr{O}}_{x}\right)$. Здесь $\widehat{\mathscr{O}}_{C}=k(C)((t))$ и $\widehat{\mathscr{O}}_{x}=k(x)[[u, t]]$. Адельное произведение этих колец приводит к трем подкольцам $\mathbb{A}_{12}, \mathbb{A}_{01}, \mathbb{A}_{02}$ в $\mathbb{A}_{X}$. Эти кольца вложены в $\mathbb{A}_{X}$ диагональным образом (в точности так же, как вложены главные адели в случае одномерной схемы). Можно сравнить структуру локальных адельных компонент в размерности 1 и 2 :
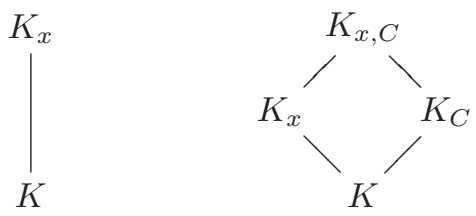

Обобщение программы Ленглендса, использующее эту теорию аделей, было предложено М. Капрановым [26] (и подробно изложено в [22]). При этом используется не обычное понятие представления, а его обобщение, связанное с 2-категориями [15].

Здесь число 2 выступает как размерность рассматриваемой схемы $X$. Имеем следующую таблицу категорий:

\begin{tabular}{|c|c|c|c|}
\hline$n=\operatorname{Dim}$ & $n$-категории & объекты & $\begin{array}{c}\text { выделенный } \\
\text { объект }\end{array}$ \\
\hline 2 & 2 -Vect $/ k$ & Vect-mod $/ k$ & Vect $/ k$ \\
\hline 1 & Vect $/ k$ & $V / k$ & $k$ \\
\hline 0 & $k$ & $A \subset k$ & $\{1\}$ \\
\hline
\end{tabular}


где $k$ - основное поле (или кольцо), $A$ - его подмножества, $V$ - векторные пространства над $k$, Vect $/ k$ - категория всех векторных пространств над $k$. Последняя является тензорной категорией, и ее можно рассматривать как категорное обобщение понятия кольца. Тогда можно ввести категории-модули Vect-mod/ $k$ над таким "кольцом" и они будут образовывать 2-категорию, объекты которой суть такие категории.

Мы видим очевидную индуктивную структуру, которая легко сопоставляется с индуктивной структурой $n$-мерных локальных полей. Кроме того, если имеется группа $G$, то можно ввести понятие ее $n$-представления. 1-представления суть обычные представления в группы автоморфизмов векторных пространств. 2-представления получаются заменой пространств на категории, а гомоморфизмов на функторы. Точнее, вместо пространства $V$ мы выбираем модульную категорию $V \in \mathrm{Ob}(2-\mathrm{Vect}-\bmod / k)$ и 2-представление $\pi$ состоит в сопоставлении элементу $g$ группы $G$ функтора $\pi(g): V \rightarrow V$, удовлетворяющего естественному обобщению условия мультипликативности. Это обобщение является частным примером общего процесса категорификации понятий и конструкций. $^{11}$

Тогда в некотором приближении соответствие Ленглендса для двумерных локальных полей $K$ выглядит по Капранову так:

$n$-мерные комплексные

(или $l$-адические) представления группы Галуа $G_{K}$

\section{неприводимые 2-представления группы $\mathrm{GL}(2 n, K)$}

и для глобального поля $K$ на схеме $X$ поле $K$ в "правой" стороне соответствия заменяется на кольцо аделей $\mathbb{A}_{X}$, а 2-представления должны удовлетворять некоторому условию автоморфности. В этих определениях необходимо уточнить входящие в них понятия и понять ряд входящих в них ограничений. Так, представления группы Галуа приходится рассматривать не любые, а с условием на простые компоненты типа однократности спектра. Кроме того, рассматриваются только поля характеристики 0 и при этом ничего не говорится о локальных полях архимедова типа. Безусловно, конструкция должна иметь место и для таких полей и для полей характеристики $p .^{12}$

\section{4. Функториальные свойства соответствия Ленглендса}

Обычное соответствие Ленглендса обладает большим количеством функториальных свойств. Теория включает два типа объектов: схему или поле и редуктивную алгебраическую группу (или, лучше сказать, корневые данные). Принцип функториальности Ленглендса [35] говорит, что происходит при замене группы. Мы ограничимся рассмотрением тех свойств, которые связаны

\footnotetext{
${ }^{11} 0$-представления мы рассмотрим позже в приложении.

${ }^{12}$ Недавно Д. В. Осипов, развивая идеи Капранова, предложил определение локального неразветвленного соответствия Ленглендса для двумерных локальных полей и группы GL $(n)$ для любого $n$.
} 
с заменой схемы ${ }^{13}$. Во-первых, это свойства, связанные с конечными расширениями рассматриваемых полей (замена базы и автоморфная индукция, или подъем (lifting)). Во-вторых, свойства, связанные с переходом от локальных полей к глобальным. Все эти свойства суть частные случаи общей функториальной конструкции обратных и прямых образов.

Первые связаны с конечными сюръективными морфизмами одномерных схем $f: X \rightarrow Y$, где $X, Y$ - или кривые (кольца целых в полях алгебраических чисел), или спектры локальных колец. Вторые возникают для морфизмов $f: X \rightarrow Y$, где $X$ - спектр локального кольца, а $Y-$ кривая. Пусть $B-$ кривая над полем $\mathbb{F}_{q}$ и $b \in B$. Имеем тогда коммутативную диаграмму

пучки $\mathscr{F}$ на $B \longrightarrow$ автоморфные формы на $\mathrm{GL}\left(\mathbb{A}_{B}\right)$

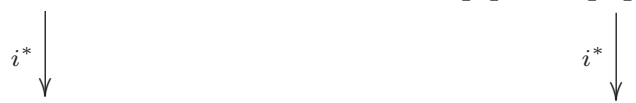

пучки $i^{*}(\mathscr{F})$ на $\operatorname{Spec}\left(\widehat{\mathscr{O}}_{b}\right) \longrightarrow$ гладкие функции на $\operatorname{GL}\left(K_{b}\right)$

где $i: \operatorname{Spec}\left(\widehat{\mathscr{O}}_{b}\right) \rightarrow B$ - каноническое вложение, левое отображение $i^{*}$ является обратным образом пучков, а правое $i^{*}$ есть взятие локальной $b$-компоненты функции на всей группе аделей (см. примеры в [18]).

Если соответствие Ленглендса существует в той или иной форме для высших размерностей, то оно должно обладать гораздо большим количеством функториальностей. Именно, пусть двумерная схема $X$ рассматривается вместе со структурным морфизмом $f: X \rightarrow B$, где $B$ - одномерная схема.

Предположим для простоты, что наши схемы суть многообразия, определенные над конечным полем. Для $l$-адических пучков (представлений групп Галуа) имеются прямые и обратные образы относительно отображения $f$. Тогда, по соответствию Ленглендса, должны существовать такие операции и для представлений групп аделей.

Не обсуждая этот вопрос в полной общности, рассмотрим частный и уже чрезвычайно интересный случай. Пусть на $X$ имеются одномерные представления группы Галуа. Им соответствуют пучки $\mathscr{F}$ ранга 1 на $X$ и их прямые образы $R^{i} f_{*}(\mathscr{F})$ на $B, i=0,1,2$.

В этой абелевой ситуации на $X$ уже имеется соответствие Ленглендса между характерами группы Галуа $G_{K}$ и характерами группы $K_{2}\left(\mathbb{A}_{X}\right)$. В силу двумерной теории полей классов (см. обзоры [11], [23], [45]) определено каноническое отображение

$$
\varphi_{X}: K_{2}\left(\mathbb{A}_{X}\right) \rightarrow G_{K}^{\mathrm{ab}},
$$

которое дает отображение групп характеров в противоположном направлении. Законы взаимности на $X$ выглядят так:

$$
\varphi_{X}\left(K_{2}\left(\mathbb{A}_{01}\right)\right)=(1), \quad \varphi_{X}\left(K_{2}\left(\mathbb{A}_{02}\right)\right)=(1) .
$$

Мы можем предположить, что на "автоморфной стороне" соответствия Ленглендса имеются операции прямого и обратного образа между характерами группы $K_{2}\left(\mathbb{A}_{X}\right)$ и автоморфными формами на кривой $B$.

\footnotetext{
13Эти два процесса не независимы. В самом деле, принцип функториальности влечет автоморфную индукцию и замену базы для конечных накрытий.
} 
Случай обратного образа является не гипотетическим, а вытекает из известных результатов высшей адельной теории. Имеется операция прямого образа абелевых групп [27], [39 $]^{14}$

$$
f_{*}: K_{2}\left(\mathbb{A}_{X}\right) \rightarrow K_{1}\left(\mathbb{A}_{B}\right) .
$$

Поскольку $K_{1}\left(\mathbb{A}_{B}\right)=\mathrm{GL}\left(1, \mathbb{A}_{B}\right)$, то получаем отображение автоморфных форм на $\operatorname{GL}\left(1, \mathbb{A}_{B}\right)$ в автоморфные формы, т. е. характеры, на $K_{2}\left(\mathbb{A}_{X}\right)$. Их "автоморфность" обеспечивается высшими законами взаимности на схеме $X$. Имеем коммутативную диаграмму

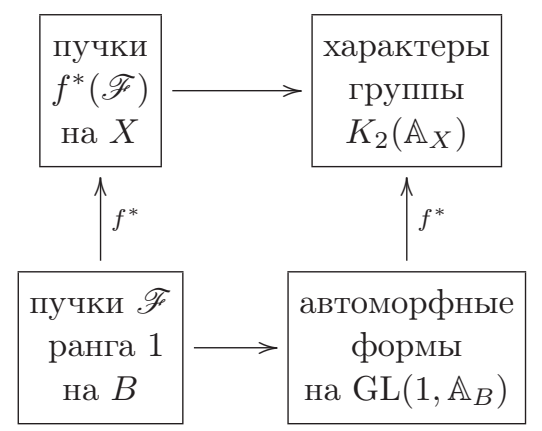

Прямой образ должен ${ }^{15}$ состоять в построении автоморфных форм $f_{i}^{*}(\chi)$, $i=0,1,2$, на $\operatorname{GL}\left(r_{i}, \mathbb{A}_{B}\right)$, отвечающей характеру $\chi$ группы $K_{2}\left(\mathbb{A}_{X}\right)$ на $X$. Должна выполняться такая коммутативная диаграмма:

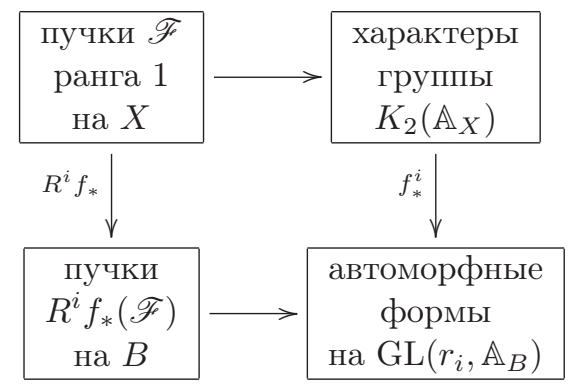

Здесь $r_{i}$ - ранг пучка $R^{i} f_{*}(\mathscr{F})$. Возможно, что вместо групп $\operatorname{GL}\left(r_{i}, \mathbb{A}_{B}\right)$ по отдельности нужно рассматривать "четные" $\mathrm{GL}\left(2, \mathbb{A}_{B}\right) \sim \mathrm{GL}\left(r_{0}, \mathbb{A}_{B}\right) \times \mathrm{GL}\left(r_{2}, \mathbb{A}_{B}\right)$

\footnotetext{
${ }^{14} \mathrm{~B}$ этих работах отображение $f$ предполагается гладким. Аналогичный гомоморфизм для дифференциальных форм был построен в [38] для негладких отображений. Совсем недавно появилась работа D. Liu [37], где был построен морфизм прямого образа для $K$-групп для негладких отображений как алгебраических, так и арифметических поверхностей.

${ }^{15}$ Мы намеренно описываем эту гипотетическую конструкцию в предварительной не совсем точной форме. Вообще говоря, соответствие Ленглендса включает в себя "справа" неприводимые автоморфные представления. Тем не менее во многих случаях можно найти в пространствах представлений порождающий вектор. Эти векторы дают автоморфные формы на группах аделей. Так, в неразветвленном случае существуют сферические векторы и неразветвленные автоморфные формы; для общих (generic) представлений можно использовать функции Уиттекера. В разделе 6 мы дадим точную формулировку в простейшем, но все же нетривиальном случае.
} 
и "нечетные" $\mathrm{GL}\left(r_{1}, \mathbb{A}_{B}\right)$ группы. Также в случае "средней" группы, отвечающей $i=1$, можно учесть монодромию, которая сохраняет кососимметрическое спаривание, и рассмотреть ортогональную группу, соответствующую симплектической группе (действия монодромии) согласно $L$-двойственности ${ }^{16}$.

ЗАМЕЧАНИЕ 2. Можно сравнить эту ситуацию со случаем числового поля, когда двумерная регулярная схема $X$ отображается на одномерную схему $B$ (кольцо целых числового поля). Мы предполагаем, что сходная конструкция существует и в этом случае. Если общий слой отображения $f$ является эллиптической кривой над $\mathbb{Q}, B=\operatorname{Spec}(\mathbb{Z})$, а $\chi$ - тривиальный характер, то автоморфная форма $f_{*}^{1}(\chi)$ есть не что иное, как адельный вариант параболической формы, отвечающей этой кривой согласно гипотезе Танияма-Вейля (ныне теореме!). В рамках программы Ленглендса ей отвечает каспидальное представление группы $\mathrm{GL}\left(2, \mathbb{A}_{B}\right)$ [33], [16]. При этом как-то оставалось в тени менее сложное представление, связанное (в наших обозначениях) с формами $f_{*}^{0}(\chi)$ и $f_{*}^{2}(\chi)$. Это формы на группе $\mathrm{GL}\left(1, \mathbb{A}_{B}\right)$, т. е. характеры $\mathbb{A}_{B}^{*}$, и, следовательно, они дают характер $\eta$ максимального тора $T$ в $G=\mathrm{GL}\left(2, \mathbb{A}_{B}\right)$. C $\eta$ (если он не равен 0) связано индуцированное представление основной серии группы $G$ и порождающий его ряд Эйзенштейна $[6 ;$ гл. 3.7, 4.5, 4.6]. Таким образом исходному характеру $\chi$ можно сопоставить два бесконечномерных представления группы $\mathrm{GL}\left(2, \mathbb{A}_{B}\right)$ : одно, “четное", принадлежащее непрерывному спектру, а другое, "нечетное" - дискретному.

Вернемся к нашей диаграмме. Поскольку мы ограничились случаем кривых над конечным полем, соответствие нижней строки построено (ДринфельдЛаффорг) и нужно “лишь" замкнуть всю диаграмму.

Представляется естественным построить сначала локальные и полулокальные прямые образы, входящие в такую диаграмму:

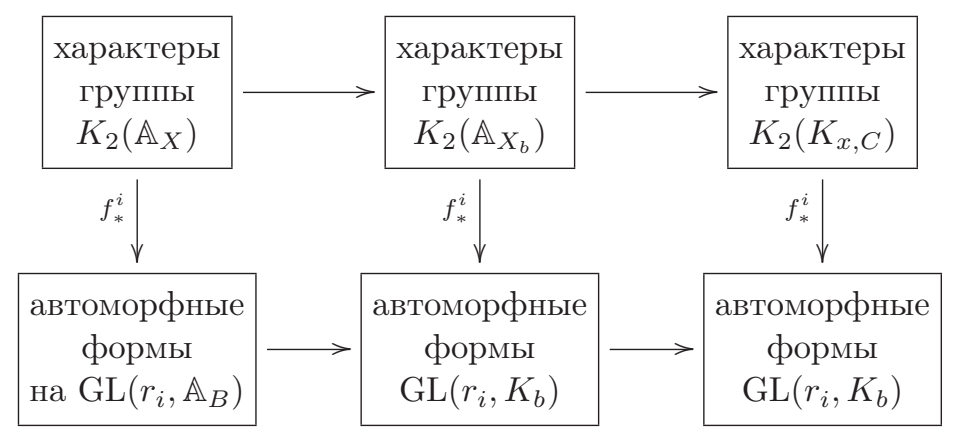

Здесь $b=f(x), C$ - кривая на $X$, проходящая через $x$ (включая слой $F_{b}$ отображения $f), X_{b}$ - двумерная схема, $X_{b}=X \times_{B} \operatorname{Spec}\left(\mathscr{O}_{b}\right)$ - инфинитезимальная окрестность слоя $f^{-1}(b)$.

Следующий шаг должен состоять в доказательстве автоморфности глобальной формы-образа на $B$, исходя из законов взаимности для характера на $K_{2}\left(\mathbb{A}_{X}\right)$. Заметим, что эти вопросы можно рассматривать совершенно независимо от

\footnotetext{
${ }^{16}$ Точнее, группу $\operatorname{GSpin}(2 g+1, \mathbb{C}), L$-двойственную группе $\operatorname{GSp}(2 g)$ симплектических подобий.
} 
того, как может быть устроено соответствие Ленглендса на двумерной схеме $X$ для пучков ранга $>1$.

Однако имеет идейное значение рассмотрение функториальных связей соответствия Ленглендса для одномерных схем (обычная теория) и схем (= конечных полей) размерности 0. Этот вопрос был поставлен мною в конце 70-х годов и обсуждался тогда с А. Бейлинсоном. Хотя было ясно, что соответствие в размерности 0 должно быть чем-то простым, придумать его нам не удалось. Когда М. Капранов прислал мне первый вариант работы [26], я предложил ему подумать над таким определением и дополнить его конструкцию для размерности два также и нульмерной ситуацией, что он и сделал в окончательном варианте. Однако в его работе не были рассмотрены функториальные свойства, связанные с сюръективными отображениями (= накрытиями) схем разной размерности.

В случае схем размерности 1 и 0 имеем такую диаграмму:

пучки $\mathscr{F}$ ранга $r$ на $X \longrightarrow$ автоморфные формы на $\operatorname{GL}\left(r, \mathbb{A}_{X}\right)$

$$
R^{i} f_{*} \downarrow
$$

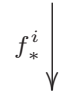

пучки $R^{i} f_{*}(\mathscr{F})$ на $\operatorname{Spec}\left(\mathbb{F}_{q}\right)$

$L$-функции

где $X$ - кривая над конечным полем $\mathbb{F}_{q}$, пучки на $\operatorname{Spec}\left(\mathbb{F}_{q}\right)$ - это векторные пространства с действием Фробениуса, а $L$-функции равны характеристическому многочлену Фробениуса. Нижняя строка относится к нульмерной ситуации и представляет собой соответствие Ленглендса для размерности нуль согласно предложению Капранова (см. обсуждение этого предложения ниже в приложении.).

Эта диаграмма должна согласовываться с гипотетической диаграммой, предлагаемой выше для морфизма схемы $X_{b}$ на $\operatorname{Spec}\left(\mathscr{O}_{b}\right)$, если в качестве кривой $X$ взять замкнутый слой $F_{b}=X \times \operatorname{Spec}(k(b))$. Исходным пунктом такого согласования служит декартова коммутативная диаграмма

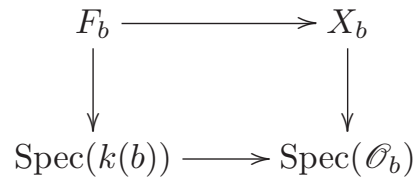

\section{5. Отношение к геометрическому соответствию Дринфельда-Ленглендса}

Рассмотрим теперь такую же картину для числового случая, когда схема $X$ отображается на одномерную схему $B$ (кольцо целых) и дополняется, согласно теории Аракелова, архимедовыми слоями $X \times_{B} \mathbb{C}$ или $X \times{ }_{B} \mathbb{R}[41]$.

Полулокальные схемы $X_{b}$ в архимедовой ситуации более не существуют, но мы должны найти аналог рассмотренных выше конструкций и в этом случае (согласно с общими принципами аналогии "числа-функции" в арифметике [41]). 
Естественно было бы предположить, что таким аналогом двумерного (!!) соответствия Ленглендса на схеме $X$ (пока лишь весьма гипотетического) является уже сформулированное и частично доказанное геометрическое соответствие Дринфельда-Ленглендса на кривой $X$, определенной над полем комплексных чисел $\mathbb{C}$, которая выступает здесь в качестве архимедова слоя. Мы обсудим эту возможность чуть позже в этом разделе.

А сейчас предположим, что в этой ситуации также имеются прямые образы и коммутативная диаграмма

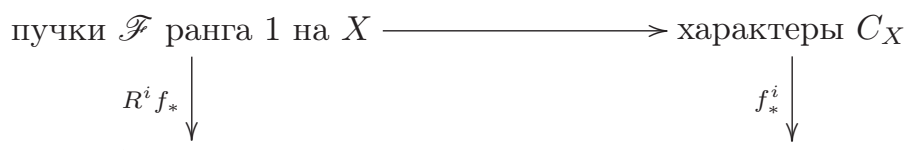

пучки $R^{i} f_{*}(\mathscr{F})$ на $\operatorname{Spec}(\mathbb{C}) \longrightarrow$ автоморфные формы на $\operatorname{GL}\left(r_{i}, \mathbb{C}\right)$

где $f: X \rightarrow \operatorname{Spec}(\mathbb{C}), C_{X}-$ подходящая формация классов (см. [2]) в теории полей классов для поля $\mathbb{C}(X)$ функций на $X$. Такие формации построены в рамках теорий полей классов для полей типа $\mathbb{C}(X)$ в 1950-е годы в работах Ж.-П. Серра, М. Хазевинкеля [47], [19] и Ю. Кавада-Дж. Тейта [28]. Они имеют следующий вид:

$$
C_{X}=\pi_{1}\left(\mathbb{A}_{X}^{*(0)} / K^{*}\right)=\pi_{1}\left(\underset{m}{\lim } J_{m}(X)\right)
$$

где $(0)$ обозначает идели степени $0, J_{m}(X)$ - обобщенные якобиевы многообразия с "модулем" $m[46]$, предел берется по всем $m$ и он является проалгебраической группой в смысле Серра [47].

Нижняя строка диаграммы (7) является хорошо известным в классической теории локальным соответствием Ленглендса для поля $\mathbb{C}$. "Пучки" на $\operatorname{Spec}(\mathbb{C})$ это векторные пространства конечной размерности над $\mathbb{C}$, снабженные структурой Ходжа. Заметим, что когомологии локальной системы (пучка) на $X$ снабжены канонической структурой Ходжа. И именно эта структура задает представление группы Вейля $W_{\mathbb{C}}$ поля $\mathbb{C}$, которая играет в этой ситуации роль группы Галуа. Имеем $W_{\mathbb{C}}=R_{\mathbb{C} / \mathbb{R}} \mathbb{G}_{m}=\mathbb{C}^{*}$. Итак, возникает следующий вопрос.

Вопрос 1. Как явным образом построить прямой образ - правую стрелку диаграммы $(7)$ ?

Но сначала надо выяснить, что же является правильным определением соответствия Ленглендса в такой ситуации. Как и выше, можно и нужно начать с локального соответствия Ленглендса, локального по $X$. В имеющейся теории Дринфельда (см. обзор [13]) рассматривается такое локальное соответствие, связанное с локальными полями вида $\mathbb{C}\left(\left(t_{P}\right)\right)$, которые отвечают точкам $P$ кривой $X\left(t_{P}-\right.$ формальный локальный параметр в точке $\left.P\right)$. Локальное соответствие должно описывать $n$-мерные представления группы, являющейся локальным вариантом группы Галуа (или фундаментальной группы в неразветвленном случае). Представляется весьма интересным уже случай абелевой группы и $n=1$. Таким образом мы приходим к вопросу о локальном аналоге 
теории полей классов для таких полей. Поскольку они являются архимедовыми вариантами двумерных локальных полей, то естественно задать такой вопрос.

Вопрос 2. Какова связь с двумерной адельной теорией полей классов?

Если $K$ - двумерное локальное поле с конечным последним полем вычетов, то теория полей классов состоит в построении канонического отображения взаимности

$$
K_{2}(K) \rightarrow G_{K}^{\mathrm{ab}}
$$

которое является локальной компонентой использованного выше глобального отображения (1). Ядро этого отображения совпадает с подгруппой делимых элементов в $K_{2}(K)$ (теорема И. Б. Фесенко, см. его обзор в [23; p. 61-74]). Примеры двумерных локальных полей суть поля $\mathbb{F}_{q}((u))((t)), \mathbb{Q}_{p}((t))$, и для них могут быть определены формации классов (весьма нетривиальным образом) и определена группа Вейля как расширение группы Галуа с помощью группы $K_{2}(K)$ [31], [1].

В случае локального поля $\mathbb{C}((t))$ с архимедовым полем вычетов имеем следующую структуру $K$-групп:

$$
K_{2} \mathbb{C}((t)) \rightarrow K_{1} \mathbb{C}=\mathbb{C}^{*}
$$

где группа $\mathbb{C}^{*}$ равна не группе Галуа поля $\mathbb{C}$, а его группе Вейля $(!)$, и можно предположить, что группа Вейля всего поля $\mathbb{C}((t))$ определяется такой же конструкцией, как и выше, с использованием группы $K_{2} \mathbb{C}((t))$. Это дает нам "левую" часть локального абелева соответствия Ленглендса для поля $\mathbb{C}((t))$. Вопрос о "правой" части соответствия может быть связан (согласно Капранову) с изучением 2-представлений. Во всяком случае получающаяся конструкция отличается от локального геометрического соответствия Дринфельда, в которое входят связности на линейных расслоениях и преобразование Фурье-Мукая. Принципиальным моментом этого отличия является то обстоятельство, что предлагаемая нами конструкция учитывает арифметическую природу основного поля $\mathbb{C}$, которая проявляется в том, что оно может быть пополнением глобального числового поля в какой-то бесконечной точке. Когда кривая, определенная над $\mathbb{C}$, является архимедовым слоем арифметической поверхности $X$, поле $\mathbb{C}$ можно отождествить с локальными пополнениями числовых полей, отвечающих горизонтальным кривым на $X$. Рис. 1 , заимствованный из [41], является простейшим примером такой ситуации.

Здесь на арифметической поверхности $X=\mathbb{P}^{1} / \operatorname{Spec}(\mathbb{Z})$ видны три горизонтальные кривые, определенные уравнениями $t=0, t=1 / 5, t=2$, где $t$ координата на общем слое $\mathbb{P}^{1} / \mathbb{R}$. Последний является кривой, лежащей над бесконечной точкой $(\infty)$ базисной “кривой” (“компактификации” схемы $\operatorname{Spec}(\mathbb{Z})$ ).

В то же время конструкция Дринфельда имеет смысл над широким классом основных полей характеристики 0 и не зависит от их природы. 


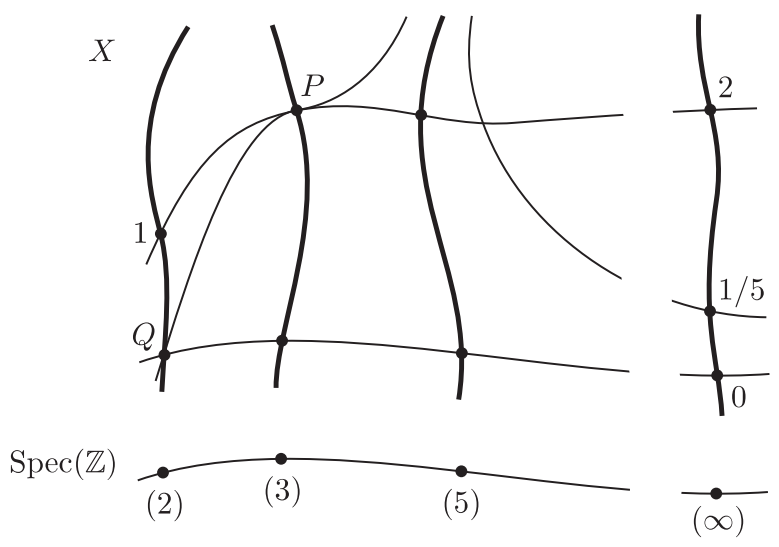

Рис. 1

ЗАмечАниЕ 3. Также должна быть аналогичная картина для кривых над $\mathbb{R}$. Здесь возникают локальные поля вида $\mathbb{R}((t))$, и для них имеем

$$
\begin{aligned}
& K_{2} \mathbb{R}[[t]] \hookrightarrow K_{2} \mathbb{R}((t)) \rightarrow K_{1} \mathbb{R}=\mathbb{R}^{*}, \\
& K_{2} \mathbb{R}[[t]] \rightarrow K_{2} \mathbb{R} \rightarrow\{ \pm 1\} .
\end{aligned}
$$

Эти разложения соответствуют неразветвленному расширению $\mathbb{C}((t)) \supset \mathbb{R}((t))$ и слабо разветвленному расширению $\mathbb{R}\left(\left(t^{1 / 2}\right)\right) \supset \mathbb{R}((t))$. Других абелевых расширений у поля $\mathbb{R}((t))$ нет. Теория полей классов для поля $K=\mathbb{R}((t))$ представляет собой канонический гомоморфизм (являющийся изоморфизмом)

$$
K_{2}(K) /\{\text { делимые элементы }\} \rightarrow \operatorname{Gal}\left(K^{\mathrm{ab}} / K\right) .
$$

Как и выше, мы предполагаем, что группа Вейля поля $\mathbb{R}((t))$ строится с использованием группы $K_{2} \mathbb{R}((t))$. Следовательно, основные поля программы Ленглендса должны быть дополнены конечными расширениями полей $\mathbb{R}(t)$ и $\mathbb{Q}_{p}(t)$, связанными с алгебраическими кривыми, определенными над полями $\mathbb{R}$ и $\mathbb{Q}_{p} . \mathrm{K}$ списку локальных полей следует добавить поля $\mathbb{R}((t))$ и $K((t))$ c $K \supset \mathbb{Q}_{p}$.

ЗАмЕчАниЕ 4. В соответствии Ленглендса фундаментальную роль играют $L$-функции. С представлениями групп Галуа (и более общо групп Вейля) связаны их $L$-функции $(L$-функции Дедекинда и Гекке для абелевых представлений и их обобщение на некоммутативный случай, введенное Э. Артином). Р. Ленглендс (и затем Р. Годеман и Э. Жаке) определили $L$-функции автоморфных представлений групп аделей редуктивных групп. Как и арифметические $L$-функции, они определяются через эйлеровское произведение локальных $L$-множителей, связанных с локальными компонентами глобального автоморфного представления (см. раздел 7).

Важнейшим свойством соответствия Ленглендса является равенство $L$-функций обеих частей соответствия (арифметических $L$-функций и $L$-функций автоморфных представлений). Глобальному равенству предшествует равенство $L$-функций и для локального соответствия. 
В случае геометрического соответствия Ленглендса, введенного Дринфельдом, по-видимому, не существует соответствующего определения $L$-функций и, следовательно, их равенства. Предполагаемая выше интерпретация геометрического соответствия подсказывает, что должны существовать $L$-функции, связанные с локальными полями $\mathbb{C}\left(\left(t_{P}\right)\right)$ точек кривой $X$, определенной над $\mathbb{C}$. Они должны входить в локальное геометрическое соответствие Ленглендса. Поскольку имеется отображение $X \rightarrow \operatorname{Spec}(\mathbb{C})$, то нужно учесть, что для поля $\mathbb{C}$ имеются $L$-функции, входящие в локальное соответствие Ленглендса для этого поля. Здесь в качестве архимедовых локальных компонент выступают гамма-функции, связанные со структурами Ходжа по Серру [48].

Прямые и обратные образы автоморфных форм, связанные с отображением $X \rightarrow \operatorname{Spec}(\mathbb{C})$, подсказывают, что гипотетические $L$-функции локальных полей $\mathbb{C}\left(\left(t_{P}\right)\right)$ точек кривой $X$ должны быть связаны с этими гамма-функциями. Пусть $z \in \mathbb{C}^{*}$ и $[z]=z /|z|,|z|_{\mathbb{C}}=|z|^{2}$. Тогда характеры группы $\mathbb{C}^{*}$ имеют вид

$$
\chi: z \mapsto[z]^{l}|z|_{\mathbb{C}}^{t}, \quad l \in \mathbb{Z}, \quad t \in \mathbb{C} .
$$

В качестве предварительного определения локальной $L$-функции, отвечающей неразветвленным расширениям в точке $P$ кривой $X$, можно предложить те же гамма-множители для характера $\chi$, что и в одномерной ситуации локального поля $\mathbb{C}[29]$ :

$$
L_{P}(s, \chi)=2(2 \pi)^{-(s+t+|l| / 2)} \Gamma(s+t+|l| / 2) .
$$

Здесь мы считаем, что неразветвленные "расширения" поля $K_{P}=\mathbb{C}\left(\left(t_{P}\right)\right)$ описываются группой Вейля его поля вычетов $\mathbb{C}$. Как мы отмечали выше, хотя поле $\mathbb{C}$ и не имеет нетривиальных расширений, роль группы Галуа в построении локального соответствия Ленглендса играет его группа Вейля.

Далее можно предположить, что глобальная $L$-функция какого-то "пучка" $\mathscr{F}$ на $X$ должна разлагаться в произведение по всем точкам кривой локальных $L$-функций.

\section{6. Гипотеза о прямом образе}

Пусть $f: X \rightarrow B$ - собственный морфизм поверхности $X$ на регулярную кривую $B$ с гладким общим слоем. Вернемся к высказанной выше гипотезе о существовании операции прямого образа из множества абелевых характеров группы $K_{2}\left(\mathbb{A}_{X}\right)$ в множество автоморфных представлений группы $\mathrm{GL}\left(\mathbb{A}_{B}\right)$ на $B$. Сформулируем ряд свойств, которым он должен удовлетворять. Отправной точкой будут для нас известные свойства прямого образа (= автоморфной индукции) для одномерного случая и неразветвленных (сферических) представлений. Перечислим их (см., например, [3], [21; предложение 4.5]).

Итак, пусть $f: B^{\prime} \rightarrow B$ - конечное неразветвленное накрытие кривых $B^{\prime}$ и $B$, имеющее степень $m$. Тогда прямой образ $f_{*}$ (автоморфная индукция АI) является отображением $\mathrm{AI}: \mathscr{A}_{\mathscr{F}^{n r}}\left(n, B^{\prime}\right) \rightarrow \mathscr{A}_{\mathscr{F}}^{n r}(n m, B)$, а обратный образ $f^{*}$ (замена базы ВC) отображением $\mathrm{BC}: \mathscr{A}_{\mathscr{F}^{n r}}(n, B) \rightarrow \mathscr{A}^{n r}\left(n, B^{\prime}\right)$. Здесь $\mathscr{A} \mathscr{F}^{n r}(n, B)$ - пространство неразветвленных автоморфных форм на $\operatorname{GL}\left(n, \mathbb{A}_{B}\right)$ :

$$
\mathscr{A} \mathscr{F}^{n r}(n, B)=\left\{\text { гладкие функции на } \operatorname{GL}(n, \mathscr{O}) \backslash \operatorname{GL}\left(n, \mathbb{A}_{B}\right) / \operatorname{GL}(n, K)\right\},
$$


где $\mathscr{O}=\prod_{x} \mathscr{O}_{x}, x \in B$, и $K=\mathbb{F}_{q}(B)$. При этом выполняются следующие свойства:

i) если $\phi \in \mathscr{A}_{\mathscr{F}^{n r}}\left(n, B^{\prime}\right)$ и $\psi \in \mathscr{A}_{\mathscr{F}^{n r}}\left(n^{\prime}, B\right)$, то справедлива формула проекции

$$
f_{*}\left(\phi \otimes f^{*}(\psi)\right)=f_{*}(\phi) \otimes \psi ;{ }^{17}
$$

ii) $f_{*}(\check{\phi})=\check{f}_{*}(\phi), f^{*}(\check{\phi})=\check{f}^{*}(\phi)$, где $\check{\phi}(g)=\phi\left({ }^{t} g^{-1}\right)$ (формула двойственности);

iii) если $n=1$, то $f_{*}(\chi)(g z)=\omega(z) f_{*}(\chi)(g)$ и $f_{*}(1)(g z)=\omega_{0}(z) f_{*}(1)(g)$, где $z \in \mathbb{A}_{B}^{*}$ - центр группы $\operatorname{GL}\left(\mathbb{A}_{B}\right), \omega, \omega_{0}$ - характеры группы $\mathbb{A}_{B}^{*}$ и $\omega \omega_{0}^{-1}=$ $\beta^{*}(\chi)$ для естественного вложения $\beta: \mathbb{A}_{B}^{*} \rightarrow \mathbb{A}_{B^{\prime}}^{*}$;

iv) для неразветвленных накрытий $f^{\prime}: B^{\prime \prime} \rightarrow B^{\prime}$ и $f: B^{\prime} \rightarrow B$ имеем

$$
\left(f^{\prime} \circ f\right)^{*}=f^{\prime *} \circ f^{*} \quad \text { и } \quad\left(f^{\prime} \circ f\right)_{*}=f_{*} \circ f_{*}^{\prime} ;
$$

v) если $k: B^{\prime} \rightarrow B^{\prime}$ и $j: B \rightarrow B$ - автоморфизмы кривых, для которых $f \circ k=j \circ f$, то выполняются формулы замены базы ${ }^{18} k^{*} \circ f_{*}=j^{*} \circ f_{*}$ и $k_{*} \circ f^{*}=j_{*} \circ f^{*}$

vi) если $\phi \in \mathscr{A} \mathscr{F}^{n r}\left(n, B^{\prime}\right)$, то $L_{\mathrm{GL}\left(n, \mathbb{A}_{B^{\prime}}\right)}(\phi)=L_{\mathrm{GL}\left(n m, \mathbb{A}_{B}\right)}\left(f_{*}(\phi)\right)$.

Определение $L$-функций см. в разделе 7 . Эти свойства должны иметь место и для гораздо более общего случая разветвленных накрытий. Насколько я знаю, в числовой ситуации они были доказаны только для циклических накрытий Галуа [3]. В геометрическом случае существование автоморфной индукции с такими свойствами следует из теоремы Лаффорга (см. сноску 20) и соответствующей конструкции для представлений групп Галуа.

Покажем, как можно получить свойство іiі) из соответствующего свойства для прямых образов $l$-адических пучков. Имеем следующее хорошо известное соотношение из теории представлений:

$$
\operatorname{Det} \rho(g) \operatorname{Det} r(g)^{-1}=\chi(\operatorname{Ver}(g))
$$

для представлений $\rho$ и $r$ некоторой группы $G$. Здесь $H \subset G-$ подгруппа конечного индекса, $\chi: H \rightarrow \mathbb{C}^{*}-$ характер, $\rho=\operatorname{ind}_{H}^{G}(\chi), r=\operatorname{ind}_{H}^{G}(1), g \in G$ и Ver: $G^{\mathrm{ab}} \rightarrow H^{\mathrm{ab}}-$ перенос (Verlagerung) (определение последнего см. в $[2$; гл. ХІІІ.2]).

В конечном неразветвленном накрытии $f: B^{\prime} \rightarrow B$ имеем вложение фундаментальных групп $\pi_{1}(B) \rightarrow \pi_{1}\left(B^{\prime}\right)$ на подгруппу конечного индекса. Применяя соотношение (8) к их представлениям, получим такую формулу для детерминанта образа локально постоянного $l$-адического пучка $\mathscr{F}$ ранга 1 на кривой $B^{\prime}$ :

$$
\text { Det } f_{*} \mathscr{F} \otimes\left(\operatorname{Det} f_{*} \mathbb{Q}_{l}\right)^{-1}=\mathrm{Nm}_{B^{\prime} / B} \mathscr{F},
$$

\footnotetext{
${ }^{17}$ Операция $\otimes$ сопоставляет формам на группах $\operatorname{GL}\left(n, \mathbb{A}_{B}\right)$ и $\operatorname{GL}\left(n^{\prime}, \mathbb{A}_{B}\right)$ форму на группе $\mathrm{GL}\left(n, \mathbb{A}_{B}\right) \times \mathrm{GL}\left(n^{\prime}, \mathbb{A}_{B}\right)$, отвечающую внешнему тензорному произведению представлений.

${ }^{18}$ Сделаем терминологическое замечание. Здесь выражение "замена базы" понимается так, как это принято в алгебраической геометрии. Это же выражение использовалось выше в смысле, принятом в теории автоморфных представлений.
} 
где $\operatorname{Det}(\mathscr{G})_{b}=\operatorname{Det}\left(\mathscr{G}_{b}\right), b \in B$, и норма пучка определяется как

$$
\mathrm{Nm}_{B^{\prime} / B} \mathscr{F}_{b}=\bigotimes_{b^{\prime} \mapsto b} \mathrm{Nm}_{k\left(b^{\prime}\right) / k(b)} \mathscr{F}_{b^{\prime}} .
$$

Чтобы получить отсюда свойство iii), нужно использовать вытекающую из теории полей классов коммутативную диаграмму

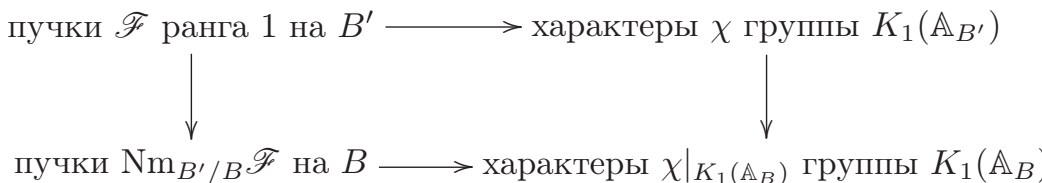

где горизонтальные стрелки суть абелевы соответствия Ленглендса, а для построения правой вертикальной стрелки использовано естественное вложение $K_{1}\left(\mathbb{A}_{B}\right) \rightarrow K_{1}\left(\mathbb{A}_{B^{\prime}}\right)$. Затем надо применить формулу для центрального характера автоморфного представления группы аделей, отвечающего $l$-адическому пучку согласно соответствию Ленглендса (теорема Лаффорга!). Именно, центральный характер равен образу относительно абелева соответствия Ленглендса пучка $\operatorname{Det} f_{*} \mathscr{F}$ ранга 1.

Когда мы переходим к двумерному случаю, число функториальных свойств увеличивается и сами свойства усложняются. Напомним, что мы рассматриваем отображения $f_{*}^{i}, i=0,1,2$, из $\mathscr{A}_{\mathscr{F}^{n r}}\left(K_{2}\left(\mathbb{A}_{X}\right)\right)$ в $\mathscr{A} \mathscr{F}^{n r}\left(\operatorname{GL}\left(r_{i}, \mathbb{A}_{B}\right)\right)$, где группа $\mathscr{A} \mathscr{F}^{n r}\left(K_{2}\left(\mathbb{A}_{X}\right)\right)$ состоит из характеров группы $K_{2}\left(\mathbb{A}_{X}\right)$, удовлетворяющих законам взаимности и тривиальных на подгруппе $K_{2}\left(\mathbb{A}_{12, X}\right)$ (условие неразветвленности). Заметим, что входящие сюда ранги $r_{i}$ зависят, вообще говоря, от $\chi$.

Гипотеза о прямом образе. Для гладких собственных морфизмов $f: X \rightarrow$ $B$ гладкой поверхности $X$ на гладкую собственную кривую $B$, определенных над конечным полем $\mathbb{F}_{q}$, существуют такие отображения $f_{*}^{i}: \mathscr{A}_{\mathscr{F}}^{n r}\left(K_{2}\left(\mathbb{A}_{X}\right)\right) \rightarrow$ $\mathscr{A} \mathscr{F}^{n r}\left(\mathrm{GL}\left(r_{i}, \mathbb{A}_{B}\right)\right), i=0,1,2$, что:

о) для характера $\chi$, тривиального на слое, $r_{0}=r_{2}=1$ и $r_{1}=2 g$; для нетривиального $\chi$ выполнены равенства $r_{0}=r_{2}=0$ и $r_{1}=2 g-2$;

i) если $\chi \in \mathscr{A}^{n r}\left(K_{2}\left(\mathbb{A}_{X}\right)\right)$ и $\eta \in \mathscr{A}^{n r}\left(K_{1}\left(\mathbb{A}_{B}\right)\right)$, то формула проекции имеет вид

$$
f_{*}^{i}\left(\chi \otimes f^{*}(\eta)\right)=f_{*}^{i}(\chi) \otimes(\eta \circ \operatorname{det}),
$$

где det: $\operatorname{GL}\left(r_{i}, \mathbb{A}_{B}\right) \rightarrow \mathbb{A}_{B}^{*}$ - определитель;

ii) для прямых образов характера $\chi \in \mathscr{A}^{n r}\left(K_{2}\left(\mathbb{A}_{X}\right)\right)$ справедлива формула двойственности

$$
\check{f}_{*}^{i}(\chi)=f_{*}^{2-i}\left(\chi^{-1} \otimes|\cdot|\right),
$$

где $|\cdot|$ - канонический характер (норма) группы $K_{2}\left(\mathbb{A}_{X}\right)($ см. [40]);

iii) $f_{*}^{i}(\chi)(g z)=\omega_{i}(z) f_{*}^{i}(\chi)(g)$ и $f_{*}^{i}(1)(g z)=\omega_{i}^{0}(z) f_{*}^{i}(1)(g)$, где $z \in \mathbb{A}_{B}^{*}$ - центр группы $\operatorname{GL}\left(\mathbb{A}_{B}\right), \omega_{i}, \omega_{i}^{0}$ - характеры группы $\mathbb{A}_{B}^{*}$ и

$$
\prod_{i} \omega_{i}^{(-1)^{i}}(z)\left(\prod_{i} \omega_{i}^{0(-1)^{i}}(z)\right)^{-1}=\chi\left(\left(\omega_{X / B}\right), \beta^{*}(z)\right),
$$


где $\beta: \mathbb{A}_{B}^{*} \rightarrow\left(\mathbb{A}_{X}^{02}\right)^{*}$ - естественное вложение, $\left(\omega_{X / B}\right) \in\left(\mathbb{A}_{X}^{01}\right)^{*}$ - идель, отвечающий относительному каноническому классу (см. [40]) и $(\cdot, \cdot): \mathbb{A}_{X}^{*} \times$ $\mathbb{A}_{X}^{*} \rightarrow K_{2}\left(\mathbb{A}_{X}\right)$ - умножение в $K$-теории;

iv) для конечного накрытия $g: B^{\prime} \rightarrow B$ степени $m$ имеем

$$
(f \circ g)_{*}^{i}(\chi)=g_{*} \circ f_{*}^{i}(\chi)
$$

на группе $\mathrm{GL}\left(r_{i} m, \mathbb{A}_{B}\right)^{19}$

v) если $k: X \rightarrow X$ и $j: B \rightarrow B$ - автоморфизмы схем, для которых $f \circ k=$ $j \circ f$, то выполняются формулы замены базы $k^{*} \circ f_{*}^{i}=j^{*} \circ f_{*}^{i}$ и $k_{*} \circ f^{*}=$ $j_{*} \circ f^{*}$

vi) $L_{X}(\chi)=\prod_{i} L_{\mathrm{GL}\left(r_{i}, \mathbb{A}_{B}\right)}\left(f_{*}^{i}(\chi)\right)^{(-1)^{i}}$

vii) также должна быть простая формула поведения при раздутиях схемы $X$ (читатель, повозись!).

Фактически, эта гипотеза может быть доказана. Именно, справедлива следующая теорема.

ТЕОрема. Существование отображений $f_{*}^{i}$, удовлетворяющих свойствам о)-vi) для гладких собственных морфизмов $f: X \rightarrow B$, вытекает из существования глобального соответствия Ленглендса для алгебрачческих кривых В над конечными полями.

Мы предпочитаем рассматривать это утверждение как гипотезу, поскольку имеющаяся тут весьма нетривиальная задача состоит в явной конструкции прямого образа, не использующей соответствие Ленглендса и вообще теорию этальных когомологий. Дадим теперь набросок того, как получить эту конструкцию, используя соответствие Ленглендса и его свойства. Обратимся к диаграмме (5) и определим отображения $f_{*}^{i}(\chi)$, используя два горизонтальных отображения этой диаграммы и левое вертикальное отображение. Верхняя стрелка, абелево двумерное соответствие Ленглендса, является биекцией, что позволяет ее обратить и тем самым начать построение прямого образа. Левая стрелка следует из теории этальных $l$-адических пучков. Наконец, самой нетривиальной частью конструкции является нижняя стрелка - глобальное соответствие Ленглендса на $B$ (теорема Лаффорга $[32]^{20}$ ).

Дадим набросок доказательств наиболее нетривиальных свойств прямого образа.

В свойстве о) мы говорим, что характер $\chi$ тривиален на слое, если для какого-то $b \in B$ его ограничение на слой $F_{b}$ тривиально на группе $\operatorname{Pic}^{0}\left(F_{b}\right)$. Свойство легко следует из известных фактов о когомологиях $l$-адических пучков.

\footnotetext{
${ }^{19}$ Композиция для конечных накрытий схемы $X$ тоже должна иметь место, но она предполагает выход за пределы группы $K_{2}\left(\mathbb{A}_{X}\right)$.

${ }^{20} \mathrm{~B}$ работе Лаффорга соответствие строится для неприводимых представлений группы Галуа и каспидальных представлений группы аделей поля функций на кривой. По-видимому, техника параболической индукции позволяет свести общий случай вполне приводимых представлений к этому. Представления группы Галуа, отвечающие пучкам $R^{i} f_{*}(\mathscr{F})$, вполне приводимы. Это вытекает из полупростоты модуля Тейта абелева многообразия, доказанной Ю. Г. Зархиным.
} 
Так как $X$ - поверхность над конечным полем, то формула двойственности іi) вытекает из теоремы двойственности для $l$-адического пучка $\mathscr{F}$ на схеме $X$, отвечающего характеру $\chi$ :

$$
\begin{aligned}
R^{i} f_{*}(\mathscr{F}) \otimes R^{2-i} f_{*}\left(\operatorname{Hom}\left(\mathscr{F}, \mathbb{Q}_{l}\right)(1)\right) & \rightarrow R^{2} f_{*}\left(\mathscr{F} \otimes \operatorname{Hom}\left(\mathscr{F}, \mathbb{Q}_{l}\right)(1)\right) \\
& \rightarrow R^{2} f_{*}\left(\mathbb{Q}_{l}\right)(1)=\mathbb{Q}_{l}
\end{aligned}
$$

и последующего применения соответствия Ленглендса.

Чтобы получить свойство іii), нужно сформулировать аналог соотношения (9) в новой ситуации. Положим

$$
\left(\mathrm{Nm}_{X / B} \mathscr{F}\right)_{b}=\bigotimes_{x \inf ^{-1}(b)} \mathscr{F}_{x}^{\nu_{x}\left(\omega_{X / B}\right)},
$$

где $\mathscr{F}$ - пучок ранга 1 на $X, b \in B, \omega_{X / B}$ - сечение относительного кокасательного расслоения и $\nu_{x}$ - нормирование в точке $x$ слоя отображения $f$. Имеем тогда

$$
\bigotimes_{i}\left(\operatorname{Det} R^{i} f_{*} \mathscr{F}\right)^{(-1)^{i}} \bigotimes_{i}\left(\operatorname{Det} R^{i} f_{*} \mathbb{Q}_{l}\right)^{(-1)^{i+1}}=\mathrm{Nm}_{X / B} \mathscr{F}
$$

Это соотношение вытекает из применения к слоям морфизма $f$ принадлежащей Делиню формулы для локально постоянного пучка $\mathscr{F}$ ранга 1 на гладкой проективной геометрически неприводимой кривой $C$ рода $g$, определенной над некоторым полем $k$. Она выглядит так:

$$
\bigotimes_{i}\left(\operatorname{DetH}^{i}(C, \mathscr{F})\right)^{(-1)^{i}}=\bigotimes_{x \in C} \mathscr{F}_{x}^{\nu_{x}(\omega)}(1-g),
$$

где $\omega$ - ненулевая дифференциальная форма на $C$ степени 1 и $\mathscr{F}(n)-$ подкрутка Тейта пучка $\mathscr{F}$ (см., например, $[40 ; \S 3.1]) .{ }^{21}$ Морфизм $f$ дает обратное отображение

$$
i: K_{1}\left(\mathbb{A}_{B}\right) \rightarrow K_{2}\left(\mathbb{A}_{X}\right), \quad i(a)=\left(a,\left(\omega_{X / B}\right)_{02}\right), \quad a \in K_{1}\left(\mathbb{A}_{B}\right),
$$

где $\left(\omega_{X / B}\right)_{02}$ есть $K_{1}\left(\mathbb{A}_{X, 02}\right)$-идель, связанный с сечением $\omega_{X / B}$ (см. $\left.[40 ; \S 2.2]\right)$. Теперь можно сформулировать и аналог диаграммы (10):

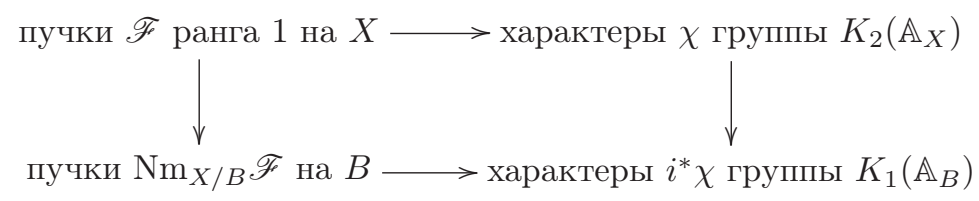

\footnotetext{
${ }^{21}$ Если $k=\mathbb{F}_{q}$ и пучок $\mathscr{F}$ отвечает характеру $\chi$ группы иделей $K_{1}\left(\mathbb{A}_{C}\right)$, то формула (11) является переформулировкой на языке $l$-адических когомологий выражения для элементарного множителя из классического функционального уравнения $L_{C}(s, \chi)=$ $\chi((\omega)) q^{s(2-2 g)} q^{g-1} L_{C}\left(1-s, \chi^{-1}\right)$ для $L$-функций $L_{C}(s, \chi)$ на кривой $C[55 ;$ гл. 7, $\S 7]$. Элементарный множитель $\chi((\omega)) q^{s(2-2 g)} q^{g-1}$ отвечает действию автоморфизма Фробениуса основного поля на одномерное $l$-адическое пространство, двойственное к пространству левой части в (11). Множитель $\chi((\omega)) q^{s(2-2 g)}$ связан с произведением $\bigotimes_{x \in C} \mathscr{F}_{x}^{\nu_{x}(\omega)}$, а подкрутка $(1-g)$ отвечает $q^{g-1}$.
} 
Затем такие же рассуждения, как и в случае конечного накрытия, дают свойство іii).

Чтобы понять свойство vi), группу $K_{2}\left(\mathbb{A}_{X}\right)$ нужно представить как адельное произведение по всем флагам $x \in C$ и вычислить следующим образом:

$$
K_{2}\left(\mathbb{A}_{X}\right) \rightarrow K_{2}\left(\mathbb{A}_{X}\right) / K_{2}\left(\mathbb{A}_{12}\right)=\bigoplus_{x \in C} \mathbb{Z}=\bigoplus_{x \in X} \bigoplus_{C \ni x} \mathbb{Z} \rightarrow \bigoplus_{x \in X} \mathbb{Z} \cdot 1_{x}
$$

Здесь последняя стрелка является суммой всех $(x, C)$-компонент вокруг данной точки $x$. Наконец, поскольку характер $\chi$ неразветвлен и автоморфен, закон взаимности влечет, что $\chi$ определен на последней группе ${ }^{22}$.

Тогда

$$
L_{X}(\chi):=\prod_{x \in|X|}\left(1-\chi\left(1_{x}\right)\right)^{-1},
$$

где $1_{x}$ - образующая локальной группы в точке $x \in X$ схемы $X . L$-функции $L_{\mathrm{GL}\left(r_{i}, \mathbb{A}_{B}\right)}\left(f_{*}^{i}(\chi)\right)$ являются стандартными $L$-функциями, связанными с представлениями, порождаемыми формами $f_{*}^{i}(\chi)$ (см. [17] и раздел 7).

Свойство vi) следует тогда из аналогичного свойства

$$
L_{X}(\mathscr{F})=\prod_{i} L_{B}\left(R^{i} f_{*}(\mathscr{F})\right)^{(-1)^{i}}
$$

для соответствующих $l$-адических пучков $\mathscr{F}$ и $R^{i} f_{*}(\mathscr{F})$ и соответствий Ленглендса на $X$ и $B$, которые влекут, что $L_{X}(\mathscr{F})=L_{X}(\chi)$ и $L_{B}\left(R^{i} f_{*}(\mathscr{F})\right)=$ $L_{\mathrm{GL}\left(r_{i}, \mathbb{A}_{B}\right)}\left(f_{*}^{i}(\chi)\right)$ для всех $i$.

ЗАмЕчАниЕ 5 . Нетрудно сформулировать локальный и полулокальный аналоги этой гипотезы (как мы уже указывали ранее) и установить, как все они связаны друг с другом. Можно даже предложить явное выражение для прямых образов в случае, когда имеется (гладкое) отображение $f: X_{b} \rightarrow \operatorname{Spec} \widehat{\mathscr{O}}_{b}$ с общим слоем рода $g$, а характер $\chi$ тривиален (или есть $s$-степень нормы $|\cdot|)$ ). Тогда $f_{*}^{0}(\chi)=|\cdot|^{s}, f_{*}^{1}(\chi)=|\cdot|^{s-1}$, а $f_{*}^{1}(\chi)$ является рядом Эйзенштейна, порождающим представление неразветвленной основной серии группы $\mathrm{GL}\left(2, K_{b}\right)$. Параметры Сатаке этого представления суть собственные значения автоморфизма Фробениуса поля $k(b)$, действующего на 1-мерных когомологиях кривой $F_{b}=X_{b} \times_{\widehat{O}_{b}} k(b)$. Можно, однако, определить их и не прибегая к $l$-адическим когомологиям, использовав метод Тейта-Ивасава на кривой $F_{b}$. Соответствующая модификация этого метода, дающая построение прямого образа, приведена в [43].

ЗАмЕчАниЕ 6. Конечно, выбор гладких морфизмов в нашей гипотезе слишком ограничителен (поскольку у нас имеется полная база $B$ ). Более разумно было бы рассмотреть, скажем, полустабильные семейства $X$ над собственной кривой $B$. В этом случае можно ожидать, что прямой образ будет давать (все еще для неразветвленных характеров $\chi$ на $X$ ) слабо разветвленные автоморфные представления $\left(\pi, V_{\pi}=\bigotimes_{b \in B} V_{\pi, b}\right)$ групп $\mathrm{GL}\left(\mathbb{A}_{B}\right)$. Если $\operatorname{dim}_{\mathbb{C}} V_{\pi, b}^{I}=1$ для

\footnotetext{
${ }^{22} \mathrm{~B}$ действительности, на ее факторгруппе $K_{2}\left(\mathbb{A}_{X}\right) / K_{2}\left(\mathbb{A}_{12}\right) K_{2}\left(\mathbb{A}_{01}\right) K_{2}\left(\mathbb{A}_{02}\right)$, которая изоморфна второй группе Чжоу. Эта группа является расширением группы $\mathbb{Z}$ с помощью конечной группы согласно теоремам конечности С. Блоха и К. Като.
} 
подгрупп Ивахори $I \subset \operatorname{GL}\left(\widehat{\mathscr{O}}_{b}\right)$ и всех точек $b$ плохой редукции, то можно сопоставить представлению $\pi$ слабо разветвленную автоморфную функцию на $\mathrm{GL}\left(\mathbb{A}_{B}\right)$. Так как для слабо разветвленных автоморфных представлений $V_{\pi}$ общего вида группы $\operatorname{GL}\left(K_{b}\right)$ имеем $\operatorname{dim}_{\mathbb{C}} V_{\pi}^{I}>1$, то, чтобы построить такую функцию, нужны дополнительные соображения. Это возможно для полустабильных эллиптических семейств [33], [16], когда в точках плохой редукции в качестве локальных компонент возникают специальные представления.

ЗАмЕчАниЕ 7. В геометрическом случае гипотеза о прямом образе вытекает из двумерной теории полей классов и соответствия Ленглендса на кривой $B$ (теорема Лаффорга). Если бы удалось определить не только абелевы автоморфные представления, но и представления некоммутативных групп аделей на схемах $X$ любой размерности (развивая, скажем, предложение Капранова) $)^{23}$, то можно было бы попытаться определить операцию прямого образа $f_{*}^{i}$, разлагая отображение $f$ в композицию проекции проективного расслоения и вложения. Это подсказывает доказательство Гротендика теоремы Римана-Роха.

\section{7. Связь с гипотезой Хассе-Вейля}

Мы предполагаем, что гипотеза о прямом образе может быть сформулирована и доказана в гораздо более общей ситуации. Именно, можно убрать условия гладкости для схем и морфизмов и рассматривать разветвленные характеры. Наконец, гипотеза безусловно может быть распространена на случай числовых полей и схем. Тогда построение прямых (и обратных) образов указанного типа давало бы возможность доказать гипотезу Хассе-Вейля [53] о мероморфном продолжении и функциональном уравнении для $L$-функций алгебраических кривых, определенных над полями алгебраических чисел (и передоказать соответствующую теорему Гротендика для $L$-функций алгебраических кривых, определенных над полями алгебраических функций с конечным полем констант, без использования $l$-адических когомологий).

Кривые с таким полем определения имеют модели (двумерные схемы $X$ со структурным морфизмом на одномерную схему $B$ - кривую над конечным полем или спектр кольца целых поля алгебраических чисел). Наличие прямого образа из $X$ на $B$ позволяет построить по характеру группы $K_{2}\left(\mathbb{A}_{X}\right)$ автоморфную форму на $B$, преобразованием Меллина которой будет интересующая нас $L$-функция. Автоморфность формы влечет, согласно общей теории, мероморфное продолжение и функциональное уравнение этой $L$-функции [54], [25], [17].

Дадим точную формулировку соответствующей теоремы для геометрического случая кривых, определенных над конечным полем. Пусть $\mathscr{A} \mathscr{F}(n, B)-$ пространство всех автоморфных форм на $\operatorname{GL}\left(n, \mathbb{A}_{B}\right)$, т. е. таких функций $F$ на $\operatorname{GL}\left(n, \mathbb{A}_{B}\right)$, что:

i) существует такой характер $\omega$ центра $Z=\mathbb{A}_{B}^{*}$ группы $\operatorname{GL}(n)$, что $F(z g)=$ $\omega(z) F(g), z \in Z, g \in \mathrm{GL}\left(n, \mathbb{A}_{B}\right)$

ii) если $\gamma \in \mathrm{GL}(n, K)$, где $K=\mathbb{F}_{q}(B)$, то $F(g \gamma)=F(g)$;

\footnotetext{
${ }^{23}$ См. сноску 12.
} 
iii) существует такая открытая компактная подгруппа $\mathbb{K}^{\prime} \subset \operatorname{GL}(n, \mathscr{O})$, где

$$
\mathscr{O}=\prod_{x} \mathscr{O}_{x}, x \in B, \text { что } F(k g)=F(g), k \in \mathbb{K}^{\prime}, g \in \operatorname{GL}\left(n, \mathbb{A}_{B}\right) .
$$

Группа $\operatorname{GL}\left(n, \mathbb{A}_{B}\right)$ действует на пространстве автоморфных форм левыми сдвигами. Гладкое представление группы $\operatorname{GL}\left(n, \mathbb{A}_{B}\right)$ называется автоморфным, если оно вкладывается как представление в пространство автоморфных форм.

Каждое неприводимое автоморфное представление $\pi$ является тензорным произведением $\bigotimes_{x \in B} \pi_{x}$ неприводимых гладких представлений локальных групп $\mathrm{GL}\left(n, K_{x}\right)$. Почти для всех $x$ представления $\pi_{x}$ являются сферическими [12], [6; гл. 3.4]. Согласно общей теории, такое представление принадлежит основной серии, т. е. получается параболической индукцией из неразветвленного характера $\chi: T \rightarrow \mathbb{C}^{*}$ максимального тора $T$ группы $\operatorname{GL}\left(n, K_{x}\right)($ см. в [6] теорему 4.6.4) для случая $n=2$, доказательство которой можно перенести на произвольное $n$ ). Такой характер задается набором $n$ комплексных чисел $z_{1}, \ldots, z_{n}$ (определенным с точностью до перестановки). Тогда можно определить локальную $L$-функцию для сферических представлений $\pi_{x}$ как

$$
L_{B}\left(s, \pi_{x}\right)=\prod_{1 \leqslant i \leqslant n}\left(1-z_{i} q^{-s}\right)^{-1}
$$

и глобальную $L$-функцию как эйлерово произведение

$$
L_{B}(s, \pi)=\prod_{x \in B} L_{B}\left(s, \pi_{x}\right) .
$$

Недостающие множители для конечного множества точек $x \in B$ определяются более сложно (см. [25; гл. 1, теорема 2.18], [16; гл. 2, теорема 8.2 и таблица А] для $n=2$ и [17; гл. 1 , теорема 3.3$]$ для произвольного $n)$.

Теорема. Пусть В - гладкая проективная кривая над конечным полем $\mathbb{F}_{q}$ и пусть $\pi$ - автоморфное представление группь $\mathrm{GL}\left(n, \mathbb{A}_{B}\right)$. Тогда:

i) эйлерово произведение (12) $L$-функции $L_{B}(s, \pi)$ сходится в правой полуплоскости $\operatorname{Re} s>n$

ii) $L_{B}(s, \pi)$ мероморфно продолжсается на всю s-плоскость и является раииональной функиией от $q^{-s}$;

iii) $L_{B}(s, \pi)=\varepsilon(s, \pi) L_{B}(n-s, \check{\pi})$, где $\varepsilon(s, \pi)$ - элементарный множитель вида $a q^{b s}, a, b \in \mathbb{R}$.

Теорема доказана для каспидальных представлений в [17; теорема 8.13]. Общий случай сводится к этому с помощью параболической индукции [24; теорема 6.2 ${ }^{24}$. Для группы GL(2) результат был получен Э. Жаке и Р. Ленглендсом в 1970 г. [25; гл. II, теорема 11.1]. Заметим, что в этих работах доказан и аналог этой теоремы для числовых полей.

Если все локальные компоненты представления $\pi$ являются сферическими, то оно порождается неразветвленной автоморфной формой и ее преобразование Меллина равно $L$-функции $L_{B}(s, \pi)$. Этот факт позволяет применить

\footnotetext{
${ }^{24}$ Мы изменили $1-s$ в формулировке из [24] на $n-s$, что достигается простой перенормировкой аргумента $L$-функции.
} 
эту теорему к автоморфным формам $f_{*}^{i}(\chi)$, возникающим как прямые образы неразветвленного автоморфного характера $\chi$ группы $K_{2}\left(\mathbb{A}_{X}\right)$ поверхности $X$, отображаемой на кривую $B$.

Рассматриваемую нами конструкцию прямого образа можно рассматривать как способ решения общей проблемы, обсужденной в [42; разд. 5.2]. Речь идет об обобщении подхода Тейта-Ивасава [50] к дзета- и $L$-функциям одномерных схем на схемы более высокой размерности (в данном случае размерности 2). Представляется, что гармонический анализ на пространстве двумерных аделей [42; разд. 2] и теория представлений дискретных групп Гейзенберга $[42$; разд. 3,4$]$ являются необходимыми техническими средствами для построения рассмотренного нами (гипотетического) прямого образа.

ЗАмечание 8 . Пусть $X-n$-мерная регулярная схема и $f: X \rightarrow B$-плоский собственный морфизм на одномерную регулярную схему $B$ с гладким общим слоем. В числовом случае схемы $X$ и $B$ необходимо рассматривать в геометрии Аракелова, пополнив их архимедовыми слоями. $n$-мерная теория полей классов предполагает существование отображения взаимности

$$
\varphi_{X}: K_{n}^{M}\left(\mathbb{A}_{X}\right) \rightarrow G_{K}^{\mathrm{ab}},
$$

где $K_{n}^{M}\left(\mathbb{A}_{X}\right)$ - милноровская часть $n$-го $K$-функтора Квиллена и $G_{K}^{\mathrm{ab}}-$ группа Галуа максимального абелева накрытия схемы $X$. Можно предположить, что гипотеза о прямом образе справедлива (с соответствующими изменениями) и для таких морфизмов и автоморфных характеров группы $K_{n}^{M}\left(\mathbb{A}_{X}\right)$. Автоморфность означает тривиальность характера на $n$ подгруппах вида

$$
K_{n}^{M}\left(\mathbb{A}_{0, \hat{1}, 2, \ldots, n}\right), \quad K_{n}^{M}\left(\mathbb{A}_{0,1, \hat{2}, \ldots, n}\right), \quad \ldots, \quad K_{n}^{M}\left(\mathbb{A}_{0,1, \ldots, \hat{n}}\right),
$$

где $\hat{i}$ означает, что $i$-й индекс опущен. Это обобщение формул (2). Так же как и выше, из такой гипотезы должна вытекать гипотеза Хассе-Вейля для схемы $X$.

Первым шагом в направлении этой гипотезы должно быть построение прямых образов для $K$-групп. Более общо, нужно построить прямые образы $f_{*}: K_{n}\left(\mathbb{A}_{X}^{\bullet}\right) \rightarrow K_{n-k}\left(\mathbb{A}_{B}^{\bullet}\right)[k]$ для собственных морфизмов $f: X \rightarrow Y$ относительной размерности $k$, полных адельных комплексов и $n-k \geqslant 0$. Мы не рассматриваем естественные условия существования таких прямых образов. Отметим только, что для замкнутых регулярных вложений отображение $f_{*}$ зависит (в отличие от случая сюръективной проекции) от выбора локальных уравнений для $Y$ в $X$. Тем не менее результирующий гомоморфизм $f_{*}$ определен однозначно, с точностью до гомотопии.

\section{8. Приложение. Нульмерное обобщение соответствия Ленглендса}

Рассмотрим более подробно аналог соответствия Ленглендса в нульмерной ситуации, т. е. для конечных полей $k$ и их расширений. Как и в случае полей более высокой размерности, теория полей классов состоит в построении канонического отображения взаимности

$$
K_{0}(k) \rightarrow G_{k}^{\mathrm{ab}}=G_{k},
$$


которое в явном виде выглядит так:

$$
\mathbb{Z} \rightarrow \widehat{\mathbb{Z}}
$$

и переводит 1 в автоморфизм Фробениуса $\operatorname{Fr}\left(\operatorname{Fr}(x)=x^{q}\right)$ для поля $k=\mathbb{F}_{q}$. Здесь $K_{0}\left(\mathbb{F}_{q}\right)$ есть $K$-группа Гротендика категории конечномерных векторных пространств и $1 \in K_{0}\left(\mathbb{F}_{q}\right)$ отвечает тривиальному одномерному пространству.

Если $k^{\prime} / k$ - конечное расширение степени $m$, то имеем коммутативные диаграммы
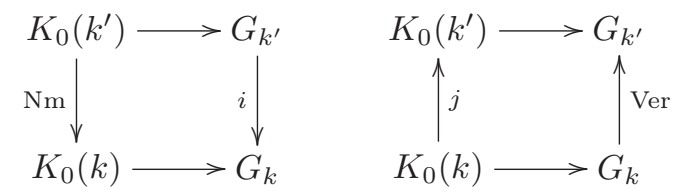

где вложения $i$ и $j$ индуцированы вложением поля $k$ в $k^{\prime}, \mathrm{Nm}$ - отображение нормы и Ver - перенос из $G_{k}^{\mathrm{ab}}=G_{k}$ в $G_{k^{\prime}}^{\mathrm{ab}}=G_{k^{\prime}}$. При этом Nm является умножением на $m$, а $j$ - изоморфизмом (групп, равных $\mathbb{Z}$ ).

Отображение (13), как и выше, приводит к отображению групп характеров (одномерных представлений) в противоположную сторону. Мы можем определить для характеров функторы прямого и обратного образа, и приведенные диаграммы приводят к их свойствам, аналогичным свойствам из предыдущего раздела.

Как мы помним, предложение Капранова состояло в том, чтобы взять $L$-функцию $L(s, \rho)$ представления Галуа $\rho$ в качестве его образа относительно 0-мерного соответствия Ленглендса. Если $\lambda_{1}, \ldots, \lambda_{n}$ - спектр представления $\rho$, то $L(s, \rho)=\operatorname{det}\left(I-\rho(\mathrm{Fr}) q^{-s}\right)=\prod_{i}\left(1-\lambda_{i} q^{-s}\right) . \quad$ С другой стороны, в случае $d$-мерных соответствий $(d=1$ или 2$)$ в автоморфной части рассматриваются $d$-представления. Можно ожидать, что нечто похожее на 0-представления должно возникнуть в правой части 0-мерного соответствия. Чтобы разобраться с этой ситуацией, приведем необходимые определения теории $d$-представлений $(d=0,1,2)$.

Это чисто алгебраическая теория, существующая независимо от арифметики. Зафиксируем группу $G$, основное поле $k$ и кардинальное число $n$. Индуктивная структура теории выглядит тогда следующим образом.

\begin{tabular}{|c|c|c|c|c|}
\hline$d$ & $\begin{array}{c}\text { пространства } \\
\text { представлений }\end{array}$ & $d$-представления & $\begin{array}{c}n=\text { размерность } \\
\text { представления }\end{array}$ & $\begin{array}{c}\text { множества } \\
\text { одномерных } \\
\text { представлений }\end{array}$ \\
\hline 2 & $V$-mod $/ k$ & $\begin{array}{c}\text { функтор } \pi(g): \\
V \text {-mod } \rightarrow V \text {-mod }\end{array}$ & $\mathrm{rk} V$-mod $/ k$ & $H^{2}\left(G, k^{*}\right)$ \\
\hline 1 & $V / k$ & $\begin{array}{c}\text { гомоморфизм } \\
\pi(g): V \rightarrow V\end{array}$ & $\operatorname{dim}_{k} V$ & $\begin{array}{c}H^{1}\left(G, k^{*}\right) \\
=\operatorname{Hom}\left(G, k^{*}\right)\end{array}$ \\
\hline 0 & $A \subset \operatorname{Sym}^{n}\left(k^{*}\right)$ & только $A$, без $\pi(g)$ & $\operatorname{deg} A=n$ & $H^{0}\left(G, k^{*}\right)=k^{*}$ \\
\hline
\end{tabular}




\begin{tabular}{|c|c|c|c|}
\hline$d$ & $\begin{array}{c}\text { отмеченный } \\
\text { объект }\end{array}$ & $\operatorname{Hom}^{\prime}$ ы & $\begin{array}{c}\text { множества } \\
\text { характеров }\end{array}$ \\
\hline 2 & $\operatorname{Vect} / k$ & $\operatorname{Func}(\mathscr{C}, \mathscr{D})$ & $\left\{g \mapsto V_{g}\right\}$ \\
\hline 1 & $k$ & $\operatorname{Hom}_{k}(V, U)$ & $\operatorname{Map}(G, k)$ \\
\hline 0 & 1 & $\left(\sum_{i} n_{i} a_{i}^{-1}\right)\left(\sum_{j} m_{j} b_{j}\right)$ & $k$ \\
\hline
\end{tabular}

Здесь $V$-mod $/ k$ - модульная категория (см. выше в разделе 3$), \operatorname{rk} V$-mod $/ k-$ ее ранг (см. [15], [26]), $V_{g}$ - векторное пространство над $k$, зависящее от $g \in G$, $V / k$ или $V$ - векторное пространство над $k$ и $\operatorname{Sym}^{n}\left(k^{*}\right)$ - множество циклов степени $n$ с неотрицательными коэффициентами. $\operatorname{Func}(\mathscr{C}, \mathscr{D})$ обозначает множество функторов из категории $\mathscr{C}$ в категорию $\mathscr{D}$, коммутирующих в категорном смысле с действием категории Vect $/ k$.

Мы видим, что для $d=0$ сама группа $G$ не участвует во всех конструкциях. Когда $d=0$, прямая сумма представлений $A$ и $B$ является суммой циклов $A$ и $B$, их тензорное произведение есть произведение Понтрягина в группе $k^{*}$, а двойственное к $A=\sum_{i} n_{i} a_{i}-$ это $\check{A}=\sum_{i} n_{i} a_{i}^{-1}$. След $\operatorname{Tr}(A)$ равен $\sum_{i} n_{i} a_{i}$, где суммирование производится в группе $k$.

Если $d=2$, то двойственным "объектом" к категории $\mathscr{C}$ будет категория функторов $\operatorname{Func}(\mathscr{C}, \operatorname{Vect} / k)$ с тем же условием, что и выше 25.

Теперь мы попытаемся применить эту небольшую теорию к (локальному) соответствию Ленглендса. Прежде всего, положим $k=\mathbb{C}$. Группой из левой части соответствия будет группа Галуа, одна и та же для всех $d$. Но справа мы видим 2-представления группы $\mathrm{GL}(2 n, K)$ для $d=2$ и 1-представления группы $\mathrm{GL}(n, K)$ для $d=1$. Можно думать, что для $d=0$ нужно рассмотреть 0 -представления группы $\mathrm{GL}(0, K)$, где последняя группа просто тривиальна. Тогда соответствие Ленглендса могло бы выглядеть так:

$$
\begin{aligned}
\operatorname{Rep}^{(n)}\left(G_{K}\right) & =\left\{n \text {-мерные полупростые представления группы } G_{K} \text { над } \mathbb{C}\right\} \\
& \stackrel{?}{\Leftrightarrow}\{\text { неприводимые } 0 \text {-представления группы } \operatorname{GL}(0, K)=(1)\},
\end{aligned}
$$

где $\rho \in \operatorname{Rep}^{(n)}\left(G_{k}\right)$ переходит в определитель $\lambda_{1} \ldots \lambda_{n}$ спектра $\lambda_{1}, \ldots, \lambda_{n}$ представления $\rho$. Мы видим, что такая конструкция не совпадает полностью с предложением Капранова. Предложенная им $L$-функция не принадлежит полю $\mathbb{C}$, как мы могли бы ожидать согласно приведенной выше таблице. Мы выбираем определитель, который принадлежит полю $\mathbb{C}$. Он, однако, удовлетворяет тому же мультипликативному свойству для точных последовательностей представлений, что и $L$-функция. Но соответствие не будет тогда биекцией в отличие от того случая, когда выбираются $L$-функции, которые суть не что иное, как характеристические многочлены автоморфизма Фробениуса. Это обстоятельство мы оставляем в качестве вопроса.

${ }^{25}$ Я благодарен участникам моего Стекловского семинара (особенно С. О. Горчинскому и Д.В. Осипову) за обсуждения, которые прояснили некоторые моменты в окончательной версии этой таблицы. 
Надо сказать, что мы встретимся еще с одной трудностью. В случае $d=1$ или 2 соответствие Ленглендса полностью согласуется с законом взаимности. Однако в случае $d=0$ мы не видим группу $K_{0}(K)$ в правой части соответствия. Эта неожиданная загадка может быть понята следующим образом.

В статье [26] Капранов оправдывает появление некоммутативной группы $\mathrm{GL}(2, K)$ в правой части абелева (!) 2-мерного соответствия следующим соотношением:

$$
K_{2}(K)=H_{2}(\mathrm{GL}(2, K), \mathbb{Z}) / H_{2}(\mathrm{GL}(1, K), \mathbb{Z}),
$$

которое является частным случаем общей теоремы А. А. Суслина о милноровской группе $K_{n}^{M}(K)$ для некоторого поля $K$. Если $n=2$, то это должно следовать из теоремы Матсумото, утверждающей, что $K_{2}^{M}(K)=K_{2}(K)=$ $\mathrm{H}_{2}(\mathrm{SL}(K), \mathbb{Z})$. Применяя этот факт, Капранов показал, что 1-мерные 1 -представления группы $K_{2}(K)$ находятся во взаимно однозначном соответствии с 1-мерными 2-представлениями группы $\mathrm{GL}(2, K)$, ограничения которых на подгруппу $\mathrm{GL}(1, K)$ тривиальны. Поскольку $K_{1}(K)=K^{*}=H_{1}(\mathrm{GL}(1, K), \mathbb{Z})$, имеем аналогичное соотношение верно и для 1 -представлений, когда $d=1$.

Для случая $d=0$ можно добавить соотношение

$$
K_{0}(K)=H_{0}(\mathrm{GL}(0, K), \mathbb{Z})=\mathbb{Z},
$$

и тогда 0-мерное соответствие Ленглендса будет выглядеть так:

$$
\begin{aligned}
\operatorname{Rep}^{(n)}\left(G_{K}\right) & =\left\{n \text {-мерные полупростые } 1 \text {-представления группы } G_{K} \text { над } \mathbb{C}\right\} \\
& \Leftrightarrow\left\{n \text {-мерные полупростые } 1 \text {-представления группы } K_{0}(K) \text { над } \mathbb{C}\right\} \\
& \Rightarrow\{1 \text {-мерные } 0 \text {-представления группы } \operatorname{GL}(0, K)=(1)\} .
\end{aligned}
$$

Такие же версии имеют место для $d=1$ или 2 , но похоже, что биекции может не быть и для $d=2$. Кроме того, необычно, что в правой части соответствия приходится рассматривать все полупростые представления, а не только неприводимые, как в одномерной ситуации.

\section{Список литературы}

[1] K. Acquista, The Weil group of a hyper-class formation, 2005, arXiv: math/0508078.

[2] E. Artin, J. Tate, Class field theory, Harvard Univ. Press, 1961.

[3] J. Arthur, L. Clozel, Simple algebras, base change, and the advanced theory of the trace formula, Ann. of Math. Stud., 120, Princeton Univ. Press, Princeton, NY, 1989, xiv $+230 \mathrm{pp}$.

[4] И.Н. Бернштейн, А.В. Зелевинский, "Представления группы $G L(n, F)$, где $F$ - локальное неархимедово поле", УМH, 31:3(189) (1976), 5-70; англ. пер.: I. N. Bernshtein, A. V. Zelevinskii, "Representations of the group $G L(n, F)$ where $F$ is a non-Archimedean local field", Russian Math. Surveys, 31:3 (1976), 1-68.

[5] L. Breen, "Tannakian categories", Motives (University of Washington, Seattle, WA, USA, July 20-August 2, 1991), Proc. Symp. Pure Math., 55, Part 1, Amer. Math. Soc., Providence, RI, 1994, 337-376.

[6] D. Bump, Automorphic forms and representations, Cambridge Stud. Adv. Math., 55, Cambridge Univ. Press, Cambridge, 1997, xiv+574 pp. 
[7] P. Cartier, "Representations of p-adic groups: a survey", Automorphic forms, representations and L-functions (Oregon State Univ., Corvallis, Ore., 1977), Proc. Sympos. Pure Math., 33, Part 1, Amer. Math. Soc., Providence, R.I., 1979, 111-155.

[8] B. Casselman, "The L-group", Class field theory - its centenary and prospect (Tokyo, 1998), Adv. Stud. Pure Math., 30, Math. Soc. Japan, Tokyo, 2001, 217-258.

[9] V. G. Drinfel'd, "Langlands'conjecture for GL(2) over functional fields", Proceedings of the International Congress of Mathematicians (Helsinki, 1978), Acad. Sci. Fennica, Helsinki, 1980, 565-574.

[10] V. G. Drinfeld, "Two-dimensional $l$-adic representations of the fundamental group of a curve over a finite field and automorphic forms on GL(2)", Amer. J. Math., 105:1 (1983), 85-114.

[11] T. Fimmel, A. N. Parshin, Introduction to the higher adelic theory, Preprint, 1999.

[12] D. Flath, "Decomposition of representations into tensor products", Automorphic forms, representations and L-functions (Oregon State Univ., Corvallis, OR, 1977), Proc. Sympos. Pure Math., 33, Part 1, Amer. Math. Soc., Providence, RI, 1979, 179-184.

[13] E. Frenkel, "Recent advances in the Langlands program", Bull. Amer. Math. Soc. (N.S.), 41:2 (2004), 151-184; 2003, arXiv: math/0303074.

[14] E. Frenkel, "Gauge theory and Langlands duality", Séminaire N. Bourbaki, v. 2008/2009, Exposés 997-1011, Astérisque, 332, Soc. Math. France, Paris, 2010, ix-x, 369-403, Exp. № 1010.

[15] N. Ganter, M. Kapranov, "Representation and character theory in 2-categories", Adv. Math., 217:5 (2008), 2268-2300; 2006, arXiv: math/0602510.

[16] S. Gelbart, "Elliptic curves and automorphic representantions", Adv. Math., 21:3 (1976), 235-292.

[17] R. Godement, H. Jacquet, Zeta functions of simple algebras, Lecture Notes in Math., 260, Springer-Verlag, Berlin-New York, 1972, viii+188 pp.

[18] M. Harris, R. Taylor, The geometry and cohomology of some simple Shimura varieties, Ann. of Math. Stud., 151, Princeton Univ. Press, Princeton, NJ, 2001, viii+276 pp.

[19] M. Hazewinkel, "Corps de classes local", Appendix to: M. Demazure, P. Gabriel, Groupes algébriques, v.I: Géométrie algébrique, généralités, groupes commutatifs, Masson, Paris; North-Holland, Amsterdam, 1970, xxvi+700 pp.

[20] G. Henniart, "Une preuve simple des conjectures de Langlands pour GL $(n)$ sur un corps p-adique", Invent. Math., 139:2 (2000), 439-455.

[21] G. Henniart, R. Herb, "Automorphic induction for GL( $n$ ) (over local nonarchimedean fields)", Duke Math. J., 78:1 (1995), 131-192.

[22] I. K. Ikeda, Multi-dimensional Langlands functoriality principle. Notes on M. M. Kapranov's work, Ser. Number Theory Appl., 3, World Scientific Publishing Co., Singapore (to appear).

[23] Invitation to higher local fields, Papers from the conference (Münster, Germany, August 29-September 5, 1999), Geom. Topol. Monogr., 3, eds. I. Fesenko, M. Kurihara, Geometry \& Topology Publications, Coventry, 2000, front matter+304 pp. (electronic); 2000, arXiv: math/0012131.

[24] H. Jacquet, "Principal $L$-functions of the linear group", Automorphic forms representations and L-functions (Oregon State Univ., Corvallis, OR, 1977), Proc. Sympos. Pure Math., 33, Part 2, Amer. Math. Soc., Providence, RI, 1979, 63-86.

[25] H. Jacquet, R. P. Langlands, Automorphic forms on GL(2), Lecture Notes in Math., 114, Springer-Verlag, Berlin-New York, 1970, vii+548 pp.; рус. пер.: Э. Жаке, Р. Ленглендс, Автоморфные формы на GL(2), Мир, М., 1973, 372 с. 
[26] M. M. Kapranov, "Analogies between the Langlands correspondence and topological quantum field theory", Functional analysis on the eve of 21 st century, v. 1, Proceedings of the conference (Rutgers University, New Brunswick, NJ, October 24-27, 1993), Progr. Math., 131, Birkhäuser, Boston, MA, 1995, 119-151.

[27] K. Kato, "Residue homomorphisms in Milnor's K-theory", Galois Groups and their Representations (Nagoya, Japan, 1981), Adv. Stud. Pure Math., 2, 1983, 153-172.

[28] Y. Kawada, J. Tate, "On the Galois cohomology of unramified extensions of function fields in one variable", Amer. J. Math., 77:2 (1955), 197-217.

[29] A.W. Knapp, "Local Langlands correspondance: the Archimedean case", Motives (University of Washington, Seattle, WA, USA, July 20-August 2, 1991), Proc. Sympos. Pure Math., 55, Part 2, ed. U. Jannsen et al., Amer. Math. Soc., Providence, RI, 1994, 393-410.

[30] H. Koch, Galoissche Theorie der p-Erweiterungen, Mathematische Monographien, 10, Springer-Verlag, Berlin-New York; VEB Deutscher Verlag der Wissenschaften, Berlin, 1970, x+161 pp.; рус. пер.: Х. Кох, Теория Галуа р-расширений, Мир, М., 1973, 199 c.

[31] Y. Koya, "A generalization of class formations by using hypercohomology", Invent. Math., 101:3 (1990), 705-715.

[32] L. Lafforgue, "Chtoucas de Drinfeld et correspondance de Langlands", Invent. Math., 147:1 (2002), 1-241.

[33] R.P. Langlands, "Problems in the theory of automorphic forms", To Salomon Bochner. In gratitude, Lectures in modern analysis and applications, v. III, Lecture Notes in Math., 170, Springer, Berlin, 1970, 18-61; pус. пер.: Р. Ленглендс, "Вопросы теории автоморфных форм”, Математика (сб. переводов), 15, № 2, Мир, M., 1971, 57-83.

[34] R. Langlands, Representations of abelian algebraic groups, 1988, 19 c. (распространялось как препринт, начиная с 1969 г.); "Representations of abelian algebraic groups", Pacific J. Math., 181:3, Spec. Issue. Olga Taussky-Todd: in memoriam (1997), 231-250.

[35] Р. Ленглендс, Функториальность и взаимность, Курс лекций, МИАН, М., сентябрь-октябрь 2011, http://publications.ias.edu/sites/default/files/lectures.pdf.

[36] G. Laumon, M. Rapoport, U. Stuhler, "D-elliptic sheaves and the Langlands correspondence", Invent. Math., 113:2 (1993), 217-338.

[37] Dongwen Liu, Tame symbols and reciprocity laws on arithmetic surfaces, 2012, arXiv: 1203.6712.

[38] M. Morrow, Grothendieck's trace map for arithmetic surfaces via residues and higher adeles, 2011, arXiv: 1101.1883.

[39] Д. В. Осипов, “Адельные конструкции прямых образов дифференциалов и символов", Матем. сб., 188:5 (1997), 59-84; англ. пер.: D. V. Osipov, "Adelic constructions of direct images of differentials and symbols", Sb. Math., 188:5 (1997), 697-723; 1998, arXiv: math/9802112.

[40] A. N. Parshin, "Chern classes, adèles and L-functions", J. Reine Angew. Math., 341 (1983), 174-192.

[41] A. N. Parshin, "Numbers as functions: the developement of an idea in the Moscow school of algebraic geometry", A lecture at the conference "Matériaux pour l'Histore des Mathématiques au XXème siècle" (Nice, 1996), Mathemathical events of the twentieth century, Springer, Berlin; Phasis, Moscow, 2006, 297-329; 2009, arXiv: 0912.3785 .

[42] A. N. Parshin, "Representations of higher adelic groups and arithmetic", Proceedings of the International Congress of Mathematicians, v. I (Hyderabad, India, August 19-27, 2010); Hindustan Book Agency, New Delhi, 2010, 362-392; 2010, arXiv: 1012.0486 . 
[43] А.Н. Паршин, "Записки о формуле Пуассона", Алгебра и анализ, 23:5 (2011), 1-54; 2010, arXiv: 1011.3392.

[44] D. Ramakrishnan, "Pure motives and automorphic forms", Motives (University of Washington, Seattle, WA, USA, July 20-August 2, 1991), Proc. Sympos. Pure Math., 55, Part 2, Amer. Math. Soc., Providence, RI, 1994, 411-446.

[45] W. Raskind, "Abelian class field theory of arithmetic schemes", K-theory and algebraic geometry: connections with quadratic forms and division algebras (July 6-24, 1992, University of California, Santa Barbara, USA), Proc. Sympos. Pure Math., 58, Part 1, eds. B. Jacob et al., Amer. Math. Soc., Providence, RI, 1995, 85-187.

[46] J.-P. Serre, Groupes algébriques et corps de classes, Publications de l'institut de mathématique de l'Université de Nancago, VII, Hermann, Paris, 1959, 202 pp.; pyc. пер.: Ж.-П. Серр, Алгебраические группы и поля классов, Мир, М., 1968, 282 с.

[47] J.-P. Serre, "Sur les corps locaux à corps résiduel algébriquement clos", Bull. Soc. Math. France, 89 (1961), 105-154.

[48] J.-P. Serre, "Facteurs locaux des fonctions zêta des variétés algébriques (Définitions et conjectures)", Theorie Nombres, Séminaire Delange-Pisot-Poitou, 11, 1969/1970:19 (1970), 1-15; рус. пер.: Ж.-П. Серр, “Локальные множители Ц-функций”, Математика (сб. переводов), 15, № 1, Мир, М., 1971, 3-13.

[49] И.Р. Шафаревич, "Поля алгебраических чисел", Proceedings of the International Congress of Mathematicians (Stockholm, 1962), Inst. Mittag-Leffler, Djursholm, 1963, $163-176$.

[50] J. Tate, Fourier analysis in number fields and Hecke's zeta functions, Thesis, Princeton University, 1950; published in: Algebraic number theory, eds. J. W.S. Cassels, A. Fröhlich, Academic Press, London-New York, 1967, 305-347.

[51] J. Tate, "Number theoretic background", Automorphic forms, representations and L-functions (Oregon State Univ., Corvallis, OR, 1977), Proc. Sympos. Pure Math., 33, Part 2, Amer. Math. Soc., Providence, RI, 1979, 3-26; pyc. пер.: Дж. Тэйт, “Теоретико-числовое введение", Автоморфные формы, представления u L-функиии, Математика. Новое в зарубежной науке, 34, Мир, М., 1984, 73-112.

[52] A. Weil, "Letter to Simone Weil (1940)", Euvres scientifiques. Collected papers, v. 1, Springer-Verlag, New York-Heidelberg, 1979, 244-256; M. H. Krieger, "A 1940 letter of André Weil on analogy in mathematics", Notices Amer. Math. Soc., 52:3 (2005), 334-341.

[53] A. Weil, "Number-theory and algebraic geometry", Proceedings of the International Congress of Mathematicians, v. 2 (Cambridge, MA, 1950), Amer. Math. Soc., Providence, RI, 1952, 90-100; рус. пер.: А. Вейль, "Теория чисел и алгебраическая геометрия", Математика (сб. переводов), 2, № 4, ИЛ, М., 1958, 49-58.

[54] A. Weil, "Zeta-functions and Mellin transforms", Algebraic geometry, Internat. Colloq. (Tata Inst. Fund. Res., Bombay, 1968), Oxford Univ. Press, London, 1969, 409-426.

[55] A. Weil, Basic number theory, Grundlehren Math. Wiss., 144, Springer-Verlag, New York, 1967, xviii+294 pp.; рус. пер.: А. Вейль, Основы теории чисел, Мир, М., $1972,408 \mathrm{c}$.

[56] Д. П. Желобенко, Гармонический анализ на полупростых комплексных группах Ли, Современные проблемы математики, Наука, М., 1974, 240 с.

А. Н. Паршин (А. N. Parshin)

Математический институт им. В. А. Стеклова РАН

E-mail: parshin@mi.ras.ru
Поступила в редакцию 30.12.2011 